\title{
A unified extragradient method for systems of hierarchical variational inequalities in a Hilbert space
}

\author{
Lu-Chuan Ceng ${ }^{1,2}$, Daya Ram Sahu ${ }^{3,4}$ and Jen-Chih Yao ${ }^{4,5 *}$
}

\section{"Correspondence:}

yaojc@kmu.edu.tw

${ }^{4}$ Center for Fundamental Science,

Kaohsiung Medical University,

Kaohsiung, 807, Taiwan

${ }^{5}$ Department of Mathematics, King

Abdulaziz University, P.O. Box 80203

Jeddah, 21589, Saudi Arabia

Full list of author information is

available at the end of the article

\begin{abstract}
In this paper, we introduce and analyze a multistep Mann-type extragradient iterative algorithm by combining Korpelevich's extragradient method, viscosity approximation method, hybrid steepest-descent method, Mann's iteration method, and the projection method. It is proven that under appropriate assumptions, the proposed algorithm converges strongly to a common element of the fixed point set of infinitely many nonexpansive mappings and a strict pseudocontraction, the solution set of finitely many generalized mixed equilibrium problems (GMEPs), the solution set of finitely many variational inclusions and the solution set of a variational inequality problem (VIP), which is just a unique solution of a system of hierarchical variational inequalities (SHVI) in a real Hilbert space. The results obtained in this paper improve and extend the corresponding results announced by many others.
\end{abstract}

MSC: 49J30; 47H09; 47J20; 49M05

Keywords: system of hierarchical variational inequalities; generalized mixed equilibrium problem; variational inclusion; variational inequality problem; multistep Mann-type extragradient method; fixed point

\section{Introduction}

Let $C$ be a nonempty closed convex subset of a real Hilbert space $H$ and $P_{C}$ be the metric projection of $H$ onto $C$. Let $S: C \rightarrow H$ be a nonlinear mapping on $C$. We denote by Fix $(S)$ the set of fixed points of $S$ and by $\mathbf{R}$ the set of all real numbers. Let $A: C \rightarrow H$ be a nonlinear mapping on $C$. We consider the following variational inequality problem (VIP): find a point $x \in C$ such that

$$
\langle A x, y-x\rangle \geq 0, \quad \forall y \in C .
$$

The solution set of VIP (1.1) is denoted by $\operatorname{VI}(C, A)$.

The VIP (1.1) was first discussed by Lions [1]. There are many applications of VIP (1.1) in various fields; see, e.g., [2-5]. It is well known that, if $A$ is a strongly monotone and Lipschitz-continuous mapping on $C$, then VIP (1.1) has a unique solution. In 1976, Korpelevich [6] proposed an iterative algorithm for solving the VIP (1.1) in Euclidean space $\mathbf{R}^{n}$ :

$$
\left\{\begin{array}{l}
y_{n}=P_{C}\left(x_{n}-\tau A x_{n}\right), \\
x_{n+1}=P_{C}\left(x_{n}-\tau A y_{n}\right), \quad \forall n \geq 0,
\end{array}\right.
$$

@2014 Ceng et al.; licensee Springer. This is an Open Access article distributed under the terms of the Creative Commons Attribution License (http://creativecommons.org/licenses/by/2.0), which permits unrestricted use, distribution, and reproduction in any medium, provided the original work is properly cited. 
with $\tau>0$ a given number, which is known as the extragradient method. The literature on the VIP is vast and Korpelevich's extragradient method has received great attention given by many authors, who improved it in various ways; see, e.g., [7-16] and references therein, to name but a few.

Let $\varphi: C \rightarrow \mathbf{R}$ be a real-valued function, $A: H \rightarrow H$ be a nonlinear mapping and $\Theta$ : $C \times C \rightarrow \mathbf{R}$ be a bifunction. In 2008, Peng and Yao [9] introduced the generalized mixed equilibrium problem (GMEP) of finding $x \in C$ such that

$$
\Theta(x, y)+\varphi(y)-\varphi(x)+\langle A x, y-x\rangle \geq 0, \quad \forall y \in C
$$

We denote the set of solutions of $\operatorname{GMEP}(1.2)$ by $\operatorname{GMEP}(\Theta, \varphi, A)$. The $\operatorname{GMEP}(1.2)$ is very general in the sense that it includes, as special cases, optimization problems, variational inequalities, minimax problems, Nash equilibrium problems in noncooperative games and others. The GMEP is further considered and studied; see, e.g., [7, 10, 14, 16-19]. In particular, if $\varphi=0$, then GMEP (1.2) reduces to the generalized equilibrium problem (GEP) which is to find $x \in C$ such that

$$
\Theta(x, y)+\langle A x, y-x\rangle \geq 0, \quad \forall y \in C .
$$

It was introduced and studied by Takahashi and Takahashi [20]. The set of solutions of GEP is denoted by $\operatorname{GEP}(\Theta, A)$.

If $A=0$, then GMEP (1.2) reduces to the mixed equilibrium problem (MEP) which is to find $x \in C$ such that

$$
\Theta(x, y)+\varphi(y)-\varphi(x) \geq 0, \quad \forall y \in C
$$

It was considered and studied in [21]. The set of solutions of $\operatorname{MEP}$ is denoted by $\operatorname{MEP}(\Theta, \varphi)$. If $\varphi=0, A=0$, then GMEP (1.2) reduces to the equilibrium problem (EP) which is to find $x \in C$ such that

$$
\Theta(x, y) \geq 0, \quad \forall y \in C .
$$

It was considered and studied in [22-24]. The set of solutions of EP is denoted by $\operatorname{EP}(\Theta)$.

On the other hand, let $B$ be a single-valued mapping of $C$ into $H$ and $R$ be a multivalued mapping with $D(R)=C$. Consider the following variational inclusion: find a point $x \in C$ such that

$$
0 \in B x+R x
$$

We denote by $\mathrm{I}(B, R)$ the solution set of the variational inclusion (1.3). In particular, if $B=R=0$, then $\mathrm{I}(B, R)=C$. If $B=0$, then problem (1.3) becomes the inclusion problem introduced by Rockafellar [25]. It is well known that problem (1.3) provides a convenient framework for the unified study of optimal solutions in many optimization related areas including mathematical programming, complementarity problems, variational inequalities, optimal control, mathematical economics, equilibria and game theory, etc. Let a set- 
valued mapping $R: D(R) \subset H \rightarrow 2^{H}$ be maximal monotone. We define the resolvent operator $J_{R, \lambda}: H \rightarrow \overline{D(R)}$ associated with $R$ and $\lambda$ as follows:

$$
J_{R, \lambda}=(I+\lambda R)^{-1}, \quad \forall x \in H,
$$

where $\lambda$ is a positive number.

In 1998, Huang [26] studied problem (1.3) in the case where $R$ is maximal monotone and $B$ is strongly monotone and Lipschitz continuous with $D(R)=C=H$. Subsequently, Zeng et al. [27] further studied problem (1.3) in the case which is more general than Huang's one [26]. Moreover, the authors [27] obtained the same strong convergence conclusion as in Huang's result [26]. In addition, the authors also gave the geometric convergence rate estimate for approximate solutions. Also, various types of iterative algorithms for solving variational inclusions have been further studied and developed; for more details, refer to $[11,19,28-30]$ and the references therein.

Let $S$ and $T$ be two nonexpansive mappings. In 2009, Yao et al. [31] considered the following hierarchical variational inequality problem (HVIP): find hierarchically a fixed point of $T$, which is a solution to the VIP for monotone mapping $I-S$; namely, find $\tilde{x} \in$ Fix $(T)$ such that

$$
\langle(I-S) \tilde{x}, p-\tilde{x}\rangle \geq 0, \quad \forall p \in \operatorname{Fix}(T) .
$$

The solution set of the hierarchical VIP (1.4) is denoted by $\Lambda$. It is not hard to check that solving the hierarchical VIP (1.4) is equivalent to the fixed point problem of the composite mapping $P_{\mathrm{Fix}(T)} S$, i.e., find $\tilde{x} \in C$ such that $\tilde{x}=P_{\mathrm{Fix}(T)} S \tilde{x}$. The authors [31] introduced and analyzed the following iterative algorithm for solving the HVIP (1.4):

$$
\left\{\begin{array}{l}
y_{n}=\beta_{n} S x_{n}+\left(1-\beta_{n}\right) x_{n} \\
x_{n+1}=\alpha_{n} V x_{n}+\left(1-\alpha_{n}\right) T y_{n}, \quad \forall n \geq 0 .
\end{array}\right.
$$

It is proved [31, Theorem 3.2] that $\left\{x_{n}\right\}$ converges strongly to $\tilde{x}=P_{\Lambda} V \tilde{x}$, which solves the hierarchical VIP:

$$
\langle(I-S) \tilde{x}, p-\tilde{x}\rangle \leq 0, \quad \forall p \in \operatorname{Fix}(T) .
$$

Very recently, Kong et al. [7] introduced and considered the following system of hierarchical variational inequalities (SHVI) (over the fixed point set of a strictly pseudocontractive mapping) with a variational inequality constraint:

to find $x^{*} \in \Xi$ such that

$$
\left\{\begin{array}{l}
\left\langle(\mu F-\gamma V) x^{*}, x-x^{*}\right\rangle \geq 0, \quad \forall x \in \operatorname{Fix}(T) \cap \operatorname{VI}(C, A), \\
\left\langle(\mu F-\gamma S) x^{*}, y-x^{*}\right\rangle \geq 0, \quad \forall y \in \operatorname{Fix}(T) \cap \operatorname{VI}(C, A) .
\end{array}\right.
$$

In particular, if $T=T_{1}$ and $A=I-T_{2}$, where $T_{i}: C \rightarrow C$ is $\xi_{i}$-strictly pseudocontractive for $i=1,2$, SHVI (1.6) reduces to the following:

to find $x^{*} \in \Omega$ such that

$$
\left\{\begin{array}{l}
\left\langle(\mu F-\gamma V) x^{*}, x-x^{*}\right\rangle \geq 0, \quad \forall x \in \operatorname{Fix}\left(T_{1}\right) \cap \operatorname{Fix}\left(T_{2}\right) \\
\left\langle(\mu F-\gamma S) x^{*}, y-x^{*}\right\rangle \geq 0, \quad \forall y \in \operatorname{Fix}\left(T_{1}\right) \cap \operatorname{Fix}\left(T_{2}\right) .
\end{array}\right.
$$


The authors in [7] proposed the following algorithm for solving SHVI (1.6) and presented its convergence analysis:

$$
\left\{\begin{array}{l}
x_{0}=x \in C \quad \text { chosen arbitrarily, } \\
y_{n}=P_{C}\left(x_{n}-v_{n} A_{n} x_{n}\right), \\
z_{n}=\beta_{n} x_{n}+\gamma_{n} P_{C}\left(x_{n}-v_{n} A_{n} y_{n}\right)+\sigma_{n} T P_{C}\left(x_{n}-v_{n} A_{n} y_{n}\right), \\
x_{n+1}=P_{C}\left[\lambda_{n} \gamma\left(\delta_{n} V x_{n}+\left(1-\delta_{n}\right) S x_{n}\right)+\left(I-\lambda_{n} \mu F\right) z_{n}\right], \quad \forall n \geq 0,
\end{array}\right.
$$

where $A_{n}=\alpha_{n} I+A$ for all $n \geq 0$. In particular, if $V \equiv 0$, then (1.8) reduces to the following iterative scheme:

$$
\left\{\begin{array}{l}
x_{0}=x \in C \quad \text { chosen arbitrarily, } \\
y_{n}=P_{C}\left(x_{n}-v_{n} A_{n} x_{n}\right), \\
z_{n}=\beta_{n} x_{n}+\gamma_{n} P_{C}\left(x_{n}-v_{n} A_{n} y_{n}\right)+\sigma_{n} T P_{C}\left(x_{n}-v_{n} A_{n} y_{n}\right), \\
x_{n+1}=P_{C}\left[\lambda_{n}\left(1-\delta_{n}\right) \gamma S x_{n}+\left(I-\lambda_{n} \mu F\right) z_{n}\right], \quad \forall n \geq 0 .
\end{array}\right.
$$

In this paper, we introduce and study the following system of hierarchical variational inequalities (SHVI) (over the fixed point set of an infinite family of nonexpansive mappings and a strictly pseudocontractive mapping) with constraints of finitely many GMEPs, finitely many variational inclusions and the VIP (1.1):

Let $M, N$ be two positive integers. Assume that

(i) $A: C \rightarrow H$ is a monotone and $L$-Lipschitzian mapping and $F: C \rightarrow H$ is $\kappa$-Lipschitzian and $\eta$-strongly monotone with positive constants $\kappa, \eta>0$ such that $0<\gamma \leq \tau$ and $0<\mu<\frac{2 \eta}{\kappa^{2}}$ where $\tau=1-\sqrt{1-\mu\left(2 \eta-\mu \kappa^{2}\right)}$

(ii) $\Theta_{k}$ is a bifunction from $C \times C$ to $\mathbf{R}$ satisfying (A1)-(A4) and $\varphi_{k}: C \rightarrow \mathbf{R} \cup\{+\infty\}$ is a proper lower semicontinuous and convex function with restriction (B1) or (B2), where $k \in\{1,2, \ldots, M\}$;

(iii) $R_{i}: C \rightarrow 2^{H}$ is a maximal monotone mapping, and $A_{k}: H \rightarrow H$ and $B_{i}: C \rightarrow H$ are $\mu_{k}$-inverse-strongly monotone and $\eta_{i}$-inverse strongly monotone, respectively, where $k \in\{1,2, \ldots, M\}$ and $i \in\{1,2, \ldots, N\}$;

(iv) $\left\{T_{n}\right\}_{n=1}^{\infty}$ is a sequence of nonexpansive self-mappings on $C, T: C \rightarrow C$ is a $\xi$-strict pseudocontraction, $S: C \rightarrow C$ is a nonexpansive mapping and $V: C \rightarrow H$ is a $\rho$-contraction with coefficient $\rho \in[0,1)$;

(v) $\Omega:=\bigcap_{n=1}^{\infty} \operatorname{Fix}\left(T_{n}\right) \cap \bigcap_{k=1}^{M} \operatorname{GMEP}\left(\Theta_{k}, \varphi_{k}, A_{k}\right) \cap \bigcap_{i=1}^{N} \mathrm{I}\left(B_{i}, R_{i}\right) \cap \operatorname{VI}(C, A) \cap \operatorname{Fix}(T) \neq \emptyset$. Then the objective is to find $x^{*} \in \Omega$ such that

$$
\left\{\begin{array}{l}
\left\langle(\mu F-\gamma V) x^{*}, x-x^{*}\right\rangle \geq 0, \quad \forall x \in \Omega, \\
\left\langle(\mu F-\gamma S) x^{*}, y-x^{*}\right\rangle \geq 0, \quad \forall y \in \Omega .
\end{array}\right.
$$

In particular, whenever $V \equiv 0$, the objective is to find $x^{*} \in \Omega$ such that

$$
\left\{\begin{array}{l}
\left\langle F x^{*}, x-x^{*}\right\rangle \geq 0, \quad \forall x \in \Omega, \\
\left\langle(\mu F-\gamma S) x^{*}, y-x^{*}\right\rangle \geq 0, \quad \forall y \in \Omega .
\end{array}\right.
$$

Motivated and inspired by the above facts, we introduce and analyze a multistep Mann-type extragradient iterative algorithm by combining Korpelevich's extragradient 
method, viscosity approximation method, hybrid steepest-descent method, Mann's iteration method and projection method. It is proven that under mild conditions, the proposed algorithm converges strongly to a common element $x^{*} \in \Omega:=\bigcap_{n=1}^{\infty} \operatorname{Fix}\left(T_{n}\right) \cap$ $\bigcap_{k=1}^{M} \operatorname{GMEP}\left(\Theta_{k}, \varphi_{k}, A_{k}\right) \cap \bigcap_{i=1}^{N} \mathrm{I}\left(B_{i}, R_{i}\right) \cap \operatorname{VI}(C, A) \cap \operatorname{Fix}(T)$ of the solution set of finitely many GMEPs, the solution set of finitely many variational inclusions, the solution set of VIP (1.1) and the fixed point set of an infinite family of nonexpansive mappings $\left\{T_{n}\right\}_{n=1}^{\infty}$ and a strict pseudocontraction $T$, which is just a unique solution of the SHVI (1.10). The results obtained in this paper improve and extend the corresponding results announced by many others. For recent related work, we refer to [32] and the references therein.

\section{Preliminaries}

Throughout this paper, we assume that $H$ is a real Hilbert space whose inner product and norm are denoted by $\langle\cdot, \cdot\rangle$ and $\|\cdot\|$, respectively.

Then we have the following inequality:

$$
\|x+y\|^{2} \leq\|x\|^{2}+2\langle y, x+y\rangle, \quad \forall x, y \in H .
$$

We write $x_{n} \rightarrow x$ to indicate that the sequence $\left\{x_{n}\right\}$ converges weakly to $x$ and $x_{n} \rightarrow x$ to indicate that the sequence $\left\{x_{n}\right\}$ converges strongly to $x$. Moreover, we use $\omega_{w}\left(x_{n}\right)$ to denote the weak $\omega$-limit set of the sequence $\left\{x_{n}\right\}$, i.e.,

$$
\omega_{w}\left(x_{n}\right):=\left\{x \in H: x_{n_{i}} \rightarrow x \text { for some subsequence }\left\{x_{n_{i}}\right\} \text { of }\left\{x_{n}\right\}\right\} .
$$

Lemma 2.1 Let $H$ be a real Hilbert space. Then the following hold:

(a) $\|x-y\|^{2}=\|x\|^{2}-\|y\|^{2}-2\langle x-y, y\rangle$ for all $x, y \in H$;

(b) $\|\lambda x+\mu y\|^{2}=\lambda\|x\|^{2}+\mu\|y\|^{2}-\lambda \mu\|x-y\|^{2}$ for all $x, y \in H$ and $\lambda, \mu \in[0,1]$ with $\lambda+\mu=1$

(c) If $\left\{x_{n}\right\}$ is a sequence in $H$ such that $x_{n} \rightarrow x$, it follows that

$$
\limsup _{n \rightarrow \infty}\left\|x_{n}-y\right\|^{2}=\limsup _{n \rightarrow \infty}\left\|x_{n}-x\right\|^{2}+\|x-y\|^{2}, \quad \forall y \in H .
$$

\subsection{Nonexpansive type mappings}

Let $C$ be a nonempty closed convex subset of $H$. The metric (or nearest point) projection from $H$ onto $C$ is the mapping $P_{C}: H \rightarrow C$ which assigns to each point $x \in H$ the unique point $P_{C} x \in C$ satisfying the property

$$
\left\|x-P_{C} x\right\|=\inf _{y \in C}\|x-y\|=: d(x, C)
$$

The following properties of projections are useful and pertinent to our purpose.

Proposition 2.1 Given any $x \in H$ and $z \in C$. Then we have the following:

(i) $z=P_{C} x \Leftrightarrow\langle x-z, y-z\rangle \leq 0, \forall y \in C$;

(ii) $z=P_{C} x \Leftrightarrow\|x-z\|^{2} \leq\|x-y\|^{2}-\|y-z\|^{2}, \forall y \in C$;

(iii) $\left\langle P_{C} x-P_{C} y, x-y\right\rangle \geq\left\|P_{C} x-P_{C} y\right\|^{2}, \forall y \in H$. 
Definition 2.1 A mapping $T: H \rightarrow H$ is said to be

(a) nonexpansive if

$$
\|T x-T y\| \leq\|x-y\|, \quad \forall x, y \in H
$$

(b) firmly nonexpansive if $2 T-I$ is nonexpansive, or equivalently, if $T$ is 1-inverse-strongly monotone (1-ism),

$$
\langle x-y, T x-T y\rangle \geq\|T x-T y\|^{2}, \quad \forall x, y \in H .
$$

Alternatively, $T$ is firmly nonexpansive if and only if $T$ can be expressed as $T=\frac{1}{2}(I+S)$, where $S: H \rightarrow H$ is nonexpansive and $I$ is the identity mapping on $H$. Note projections are firmly nonexpansive.

Definition 2.2 A mapping $T: H \rightarrow H$ is said to be an averaged mapping if it can be written as the average of the identity $I$ and a nonexpansive mapping, that is, $T \equiv(1-\alpha) I+\alpha S$, where $\alpha \in(0,1)$ and $S: H \rightarrow H$ is nonexpansive.

Proposition 2.2 ([33]) Let $T: H \rightarrow H$ be a given mapping. Then:

(i) $T$ is nonexpansive if and only if the complement $I-T$ is $\frac{1}{2}$-ism.

(ii) If $T$ is $v$-ism, then for $\gamma>0, \gamma T$ is $\frac{v}{\gamma}$-ism.

(iii) $T$ is averaged if and only if the complement $I-T$ is $v$-ism for some $v>1 / 2$. Indeed, for $\alpha \in(0,1), T$ is $\alpha$-averaged if and only if $I-T$ is $\frac{1}{2 \alpha}$-ism.

Proposition $2.3([33,34])$ Let $S, T, V: H \rightarrow H$ be given operators.

(i) If $T=(1-\alpha) S+\alpha V$ for some $\alpha \in(0,1)$ and if $S$ is averaged and $V$ is nonexpansive, then $T$ is averaged.

(ii) $T$ is firmly nonexpansive if and only if the complement $I-T$ is firmly nonexpansive.

(iii) If $T=(1-\alpha) S+\alpha V$ for some $\alpha \in(0,1)$ and if $S$ is firmly nonexpansive and $V$ is nonexpansive, then $T$ is averaged.

(iv) The composite of finitely many averaged mappings is averaged. That is, if each of the mappings $\left\{T_{i}\right\}_{i=1}^{N}$ is averaged, then so is the composite $T_{1} \cdots T_{N}$. In particular, if $T_{1}$ is $\alpha_{1}$-averaged and $T_{2}$ is $\alpha_{2}$-averaged, where $\alpha_{1}, \alpha_{2} \in(0,1)$, then the composite $T_{1} T_{2}$ is $\alpha$-averaged, where $\alpha=\alpha_{1}+\alpha_{2}-\alpha_{1} \alpha_{2}$.

(v) If the mappings $\left\{T_{i}\right\}_{i=1}^{N}$ are averaged and have a common fixed point, then

$$
\bigcap_{i=1}^{N} \operatorname{Fix}\left(T_{i}\right)=\operatorname{Fix}\left(T_{1} \cdots T_{N}\right)
$$

We need some facts and tools which are listed as lemmas below.

Lemma 2.2 ([35, Demiclosedness principle]) Let C be a nonempty closed convex subset of a real Hilbert space H. Let $S$ be a nonexpansive self-mapping on $C$. Then $I-S$ is demiclosed at 0 . 
Let $\left\{T_{n}\right\}_{n=1}^{\infty}$ be an infinite family of nonexpansive self-mappings on $C$ and $\left\{\lambda_{n}\right\}_{n=1}^{\infty}$ be a sequence in $[0,1]$. For any $n \geq 1$, define a mapping $W_{n}$ on $C$ as follows:

$$
\left\{\begin{array}{l}
U_{n, n+1}=I \\
U_{n, n}=\lambda_{n} T_{n} U_{n, n+1}+\left(1-\lambda_{n}\right) I \\
U_{n, n-1}=\lambda_{n-1} T_{n-1} U_{n, n}+\left(1-\lambda_{n-1}\right) I \\
\ldots, \\
U_{n, k}=\lambda_{k} T_{k} U_{n, k+1}+\left(1-\lambda_{k}\right) I, \\
U_{n, k-1}=\lambda_{k-1} T_{k-1} U_{n, k}+\left(1-\lambda_{k-1}\right) I \\
\ldots, \\
U_{n, 2}=\lambda_{2} T_{2} U_{n, 3}+\left(1-\lambda_{2}\right) I \\
W_{n}=U_{n, 1}=\lambda_{1} T_{1} U_{n, 2}+\left(1-\lambda_{1}\right) I
\end{array}\right.
$$

Such a mapping $W_{n}$ is called the $W$-mapping generated by $T_{n}, T_{n-1}, \ldots, T_{1}$ and $\lambda_{n}, \lambda_{n-1}$, $\ldots, \lambda_{1}$.

Lemma 2.3 ([36]) Let $C$ be a nonempty closed convex subset of a real Hilbert space H. Let $\left\{T_{n}\right\}_{n=1}^{\infty}$ be a sequence of nonexpansive self-mappings on $C$ such that $\bigcap_{n=1}^{\infty} \operatorname{Fix}\left(T_{n}\right) \neq \emptyset$ and let $\left\{\lambda_{n}\right\}_{n=1}^{\infty}$ be a sequence in $(0, b]$ for some $b \in(0,1)$. Then, for every $x \in C$ and $k \geq 1$ the limit $\lim _{n \rightarrow \infty} U_{n, k} x$ exists, where $U_{n, k}$ is defined as in (2.1).

Remark 2.1 ([37, Remark 3.1]) It can be known from Lemma 2.4 that if $D$ is a nonempty bounded subset of $C$, then for $\epsilon>0$ there exists $n_{0} \geq k$ such that $\sup _{x \in D}\left\|U_{n, k} x-U_{k} x\right\| \leq \epsilon$ for all $n>n_{0}$.

Remark 2.2 ([37, Remark 3.2]) Utilizing Lemma 2.4, we define a mapping $W: C \rightarrow C$ as follows:

$$
W x=\lim _{n \rightarrow \infty} W_{n} x=\lim _{n \rightarrow \infty} U_{n, 1} x, \quad \forall x \in C .
$$

Such a $W$ is called the $W$-mapping generated by $T_{1}, T_{2}, \ldots$ and $\lambda_{1}, \lambda_{2}, \ldots$ Since $W_{n}$ is nonexpansive, $W: C \rightarrow C$ is also nonexpansive. For a bounded sequence $\left\{x_{n}\right\}$ in $C$, we put $D=\left\{x_{n}: n \geq 1\right\}$. Hence, it is clear from Remark 2.1 that for an arbitrary $\epsilon>0$, there exists $N_{0} \geq 1$ such that for all $n>N_{0}$

$$
\left\|W_{n} x_{n}-W x_{n}\right\|=\left\|U_{n, 1} x_{n}-U_{1} x_{n}\right\| \leq \sup _{x \in D}\left\|U_{n, 1} x-U_{1} x\right\| \leq \epsilon
$$

This implies that $\lim _{n \rightarrow \infty}\left\|W_{n} x_{n}-W x_{n}\right\|=0$.

Lemma 2.4 ([36]) Let $C$ be a nonempty closed convex subset of a real Hilbert space $H$. Let $\left\{T_{n}\right\}_{n=1}^{\infty}$ be a sequence of nonexpansive self-mappings on $C$ such that $\bigcap_{n=1}^{\infty} \operatorname{Fix}\left(T_{n}\right) \neq \emptyset$, and let $\left\{\lambda_{n}\right\}_{n=1}^{\infty}$ be a sequence in $(0, b]$ for some $b \in(0,1)$. Then $\operatorname{Fix}(W)=\bigcap_{n=1}^{\infty} \operatorname{Fix}\left(T_{n}\right)$.

It is clear that, in a real Hilbert space $H, T: C \rightarrow C$ is $\xi$-strictly pseudocontractive if and only if the following inequality holds:

$$
\langle T x-T y, x-y\rangle \leq\|x-y\|^{2}-\frac{1-\xi}{2}\|(I-T) x-(I-T) y\|^{2}, \quad \forall x, y \in C .
$$


This immediately implies that if $T$ is a $\xi$-strictly pseudocontractive mapping, then $I-T$ is $\frac{1-\xi}{2}$-inverse-strongly monotone; for further detail, we refer to [38] and the references therein. It is well known that the class of strict pseudocontractions strictly includes the class of nonexpansive mappings and that the class of pseudocontractions strictly includes the class of strict pseudocontractions. In addition, for the extension of strict pseudocontractions, please the reader refer to [39] and the references therein.

Proposition 2.4 ([38, Proposition 2.1]) Let $C$ be a nonempty closed convex subset of a real Hilbert space $H$ and $T: C \rightarrow C$ be a mapping.

(i) If $T$ is a $\xi$-strictly pseudocontractive mapping, then $T$ satisfies the Lipschitzian condition

$$
\|T x-T y\| \leq \frac{1+\xi}{1-\xi}\|x-y\|, \quad \forall x, y \in C .
$$

(ii) If $T$ is a $\xi$-strictly pseudocontractive mapping, then the mapping $I-T$ is demiclosed at 0 , that is, if $\left\{x_{n}\right\}$ is a sequence in $C$ such that $x_{n} \rightarrow \tilde{x}$ and $(I-T) x_{n} \rightarrow 0$, then $(I-T) \tilde{x}=0$.

(iii) If $T$ is $\xi$-(quasi-)strict pseudocontraction, then the fixed point set $\operatorname{Fix}(T)$ of $T$ is closed and convex so that the projection $P_{\mathrm{Fix}(T)}$ is well defined.

Proposition 2.5 ([40]) Let $C$ be a nonempty closed convex subset of a real Hilbert space $H$. Let $T: C \rightarrow C$ be a $\xi$-strictly pseudocontractive mapping. Let $\gamma$ and $\delta$ be two nonnegative real numbers such that $(\gamma+\delta) \xi \leq \gamma$. Then

$$
\|\gamma(x-y)+\delta(T x-T y)\| \leq(\gamma+\delta)\|x-y\|, \quad \forall x, y \in C .
$$

\subsection{Mixed equilibrium problems}

We list some elementary conclusions for the MEP.

It was assumed in [9] that $\Theta: C \times C \rightarrow \mathbf{R}$ is a bifunction satisfying conditions (A1)-(A4) and $\varphi: C \rightarrow \mathbf{R}$ is a lower semicontinuous and convex function with restriction (B1) or (B2), where

(A1) $\Theta(x, x)=0$ for all $x \in C$;

(A2) $\Theta$ is monotone, i.e., $\Theta(x, y)+\Theta(y, x) \leq 0$ for any $x, y \in C$;

(A3) $\Theta$ is upper-hemicontinuous, i.e., for each $x, y, z \in C$,

$$
\limsup _{t \rightarrow 0^{+}} \Theta(t z+(1-t) x, y) \leq \Theta(x, y)
$$

(A4) $\Theta(x, \cdot)$ is convex and lower semicontinuous for each $x \in C$;

(B1) for each $x \in H$ and $r>0$, there exists a bounded subset $D_{x} \subset C$ and $y_{x} \in C$ such that for any $z \in C \backslash D_{x}$,

$$
\Theta\left(z, y_{x}\right)+\varphi\left(y_{x}\right)-\varphi(z)+\frac{1}{r}\left\langle y_{x}-z, z-x\right\rangle<0
$$

(B2) $C$ is a bounded set.

Proposition 2.6 ([21]) Assume that $\Theta: C \times C \rightarrow \mathbf{R}$ satisfies (A1)-(A4) and let $\varphi: C \rightarrow \mathbf{R}$ be a proper lower semicontinuous and convex function. Assume that either (B1) or (B2) 
holds. For $r>0$ and $x \in H$, define a mapping $T_{r}^{(\Theta, \varphi)}: H \rightarrow C$ as follows:

$$
T_{r}^{(\Theta, \varphi)}(x)=\left\{z \in C: \Theta(z, y)+\varphi(y)-\varphi(z)+\frac{1}{r}\langle y-z, z-x\rangle \geq 0, \forall y \in C\right\}
$$

for all $x \in H$. Then the following hold:

(i) for each $x \in H, T_{r}^{(\Theta, \varphi)}(x)$ is nonempty and single-valued;

(ii) $T_{r}^{(\Theta, \varphi)}$ is firmly nonexpansive, that is, for any $x, y \in H$,

$$
\left\|T_{r}^{(\Theta, \varphi)} x-T_{r}^{(\Theta, \varphi)} y\right\|^{2} \leq\left\langle T_{r}^{(\Theta, \varphi)} x-T_{r}^{(\Theta, \varphi)} y, x-y\right|
$$

(iii) $\operatorname{Fix}\left(T_{r}^{(\Theta, \varphi)}\right)=\operatorname{MEP}(\Theta, \varphi)$;

(iv) $\operatorname{MEP}(\Theta, \varphi)$ is closed and convex;

(v) $\left\|T_{s}^{(\Theta, \varphi)} x-T_{t}^{(\Theta, \varphi)} x\right\|^{2} \leq \frac{s-t}{s}\left\langle T_{s}^{(\Theta, \varphi)} x-T_{t}^{(\Theta, \varphi)} x, T_{s}^{(\Theta, \varphi)} x-x\right\rangle$ for all $s, t>0$ and $x \in H$.

\subsection{Monotone operators}

Definition 2.3 Let $T$ be a nonlinear operator with the domain $D(T) \subseteq H$ and the range $R(T) \subseteq H$. Then $T$ is said to be

(i) monotone if

$$
\langle T x-T y, x-y\rangle \geq 0, \quad \forall x, y \in D(T)
$$

(ii) $\beta$-strongly monotone if there exists a constant $\beta>0$ such that

$$
\langle T x-T y, x-y\rangle \geq \eta\|x-y\|^{2}, \quad \forall x, y \in D(T)
$$

(iii) $v$-inverse-strongly monotone if there exists a constant $v>0$ such that

$$
\langle T x-T y, x-y\rangle \geq v\|T x-T y\|^{2}, \quad \forall x, y \in D(T) .
$$

It can easily be seen that if $T$ is nonexpansive, then $I-T$ is monotone. It is also easy to see that the projection $P_{C}$ is 1-ism. Inverse strongly monotone (also referred to as co-coercive) operators have been applied widely in solving practical problems in various fields, for instance, in traffic assignment problems; see [41, 42]. On the other hand, it is obvious that if $A$ is $\zeta$-inverse-strongly monotone, then $A$ is monotone and $\frac{1}{\zeta}$-Lipschitz continuous. Moreover, we also have, for all $u, v \in C$ and $\lambda>0$,

$$
\begin{aligned}
\|(I-\lambda A) u-(I-\lambda A) v\|^{2} & =\|(u-v)-\lambda(A u-A v)\|^{2} \\
& =\|u-v\|^{2}-2 \lambda\langle A u-A v, u-v\rangle+\lambda^{2}\|A u-A v\|^{2} \\
& \leq\|u-v\|^{2}+\lambda(\lambda-2 \zeta)\|A u-A v\|^{2} .
\end{aligned}
$$

So, if $\lambda \leq 2 \zeta$, then $I-\lambda A$ is a nonexpansive mapping from $C$ to $H$.

Let $C$ be a nonempty closed convex subset of a real Hilbert space $H$. We introduce some notations. Let $\lambda$ be a number in $(0,1]$ and let $\mu>0$. Associated with a nonexpansive mapping $T: C \rightarrow C$, we define the mapping $T^{\lambda}: C \rightarrow H$ by

$$
T^{\lambda} x:=T x-\lambda \mu F(T x), \quad \forall x \in C,
$$


where $F: C \rightarrow H$ is an operator such that, for some positive constants $\kappa, \eta>0, F$ is $\kappa$-Lipschitzian and $\eta$-strongly monotone on $C$; that is, $F$ satisfies the conditions:

$$
\|F x-F y\| \leq \kappa\|x-y\| \quad \text { and } \quad\langle F x-F y, x-y\rangle \geq \eta\|x-y\|^{2}, \quad \forall x, y \in C .
$$

Lemma 2.5 (see [43, Lemma 3.1]) $T^{\lambda}$ is a contraction provided $0<\mu<\frac{2 \eta}{\kappa^{2}}$; that is,

$$
\left\|T^{\lambda} x-T^{\lambda} y\right\| \leq(1-\lambda \tau)\|x-y\|, \quad \forall x, y \in C,
$$

where $\tau=1-\sqrt{1-\mu\left(2 \eta-\mu \kappa^{2}\right)} \in(0,1]$.

Remark 2.3 (i) Since $F$ is $\kappa$-Lipschitzian and $\eta$-strongly monotone on $C$, we get $0<$ $\eta \leq \kappa$. Hence, whenever $0<\mu<\frac{2 \eta}{\kappa^{2}}$, we have $0<1-\sqrt{1-2 \mu \eta+\mu^{2} \kappa^{2}} \leq 1$. So, $\tau=1-$ $\sqrt{1-\mu\left(2 \eta-\mu \kappa^{2}\right)} \in(0,1]$.

(ii) In Lemma 2.5, put $F=\frac{1}{2} I$ and $\mu=2$. Then we know that $\kappa=\eta=\frac{1}{2}, 0<\mu=2<\frac{2 \eta}{\kappa^{2}}=4$ and

$$
\tau=1-\sqrt{1-\mu\left(2 \eta-\mu \kappa^{2}\right)}=1-\sqrt{1-2\left(2 \times \frac{1}{2}-2 \times\left(\frac{1}{2}\right)^{2}\right)}=1 .
$$

Lemma 2.6 Let $A: C \rightarrow H$ be a monotone mapping. The characterization of the projection (see Proposition 2.1(i)) implies

$$
u \in \operatorname{VI}(C, A) \quad \Leftrightarrow \quad u=P_{C}(u-\lambda A u), \quad \forall \lambda>0 .
$$

Finally, recall that a set-valued mapping $\widetilde{T}: D(\widetilde{T}) \subseteq H \rightarrow 2^{H}$ is called monotone if for all $x, y \in D(\widetilde{T}), f \in \widetilde{T} x$ and $g \in \widetilde{T} y$ imply

$$
\langle f-g, x-y\rangle \geq 0
$$

A set-valued mapping $\widetilde{T}$ is called maximal monotone if $\widetilde{T}$ is monotone and $(I+\lambda \widetilde{T}) D(\widetilde{T})=$ $H$ for each $\lambda>0$. We denote by $G(\widetilde{T})$ the graph of $\widetilde{T}$. It is well known that a monotone mapping $\widetilde{T}$ is maximal if and only if, for $(x, f) \in H \times H,\langle f-g, x-y\rangle \geq 0$ for every $(y, g) \in$ $G(\widetilde{T})$ implies $f \in \widetilde{T} x$. Next we provide an example to illustrate the concept of maximal monotone mapping.

Let $A: C \rightarrow H$ be a monotone, $k$-Lipschitz-continuous mapping and let $N_{C} v$ be the normal cone to $C$ at $v \in C$, i.e.,

$$
N_{C}(v)=\{u \in H:\langle v-x, u\rangle \geq 0, \forall x \in C\} .
$$

Define

$$
\widetilde{T} v= \begin{cases}A v+N_{C}(v), & \text { if } v \in C, \\ \emptyset, & \text { if } v \notin C .\end{cases}
$$

Then $\widetilde{T}$ is maximal monotone (see [25]) such that

$$
0 \in \widetilde{T} v \quad \Leftrightarrow \quad v \in \mathrm{VI}(C, A) .
$$


Let $R: D(R) \subseteq H \rightarrow 2^{H}$ be a maximal monotone mapping. Let $\lambda, \mu>0$ be two positive numbers.

Lemma 2.7 (see [44]) We have the resolvent identity

$$
J_{R, \lambda} x=J_{R, \mu}\left(\frac{\mu}{\lambda} x+\left(1-\frac{\mu}{\lambda}\right) J_{R, \lambda} x\right), \quad \forall x \in H
$$

In terms of Huang [26] (see also [27]), we have the following property for the resolvent operator $J_{R, \lambda}: H \rightarrow \overline{D(R)}$.

Lemma 2.8 $J_{R, \lambda}$ is single-valued and firmly nonexpansive, i.e.,

$$
\left\langle J_{R, \lambda} x-J_{R, \lambda} y, x-y\right\rangle \geq\left\|J_{R, \lambda} x-J_{R, \lambda} y\right\|^{2}, \quad \forall x, y \in H .
$$

Consequently, $J_{R, \lambda}$ is nonexpansive and monotone.

Lemma 2.9 ([11]) Let $R$ be a maximal monotone mapping with $D(R)=C$. Then for any given $\lambda>0, u \in C$ is a solution of problem (1.3) if and only if $u \in C$ satisfies

$$
u=J_{R, \lambda}(u-\lambda B u) .
$$

Lemma 2.10 ([27]) Let $R$ be a maximal monotone mapping with $D(R)=C$ and let $B: C \rightarrow$ $H$ be a strongly monotone, continuous and single-valued mapping. Then for each $z \in H$, the equation $z \in(B+\lambda R) x$ has a unique solution $x_{\lambda}$ for $\lambda>0$.

Lemma 2.11 ([11]) Let $R$ be a maximal monotone mapping with $D(R)=C$ and $B: C \rightarrow H$ be a monotone, continuous and single-valued mapping. Then $(I+\lambda(R+B)) C=H$ for each $\lambda>0$. In this case, $R+B$ is maximal monotone.

\subsection{Technical lemmas}

The following lemma plays a key role in proving strong convergence of the sequences generated by our algorithms.

Lemma 2.12 ([45]) Let $\left\{a_{n}\right\}$ be a sequence of nonnegative real numbers satisfying the property:

$$
a_{n+1} \leq\left(1-s_{n}\right) a_{n}+s_{n} b_{n}+t_{n}, \quad \forall n \geq 1,
$$

where $\left\{s_{n}\right\} \subset(0,1]$ and $\left\{b_{n}\right\}$ are such that

(i) $\sum_{n=1}^{\infty} s_{n}=\infty$;

(ii) either $\limsup _{n \rightarrow \infty} b_{n} \leq 0$ or $\sum_{n=1}^{\infty}\left|s_{n} b_{n}\right|<\infty$;

(iii) $\sum_{n=1}^{\infty} t_{n}<\infty$ where $t_{n} \geq 0$, for all $n \geq 1$.

Then $\lim _{n \rightarrow \infty} a_{n}=0$.

Lemma 2.13 ([46]) Let $\left\{\alpha_{n}\right\}$ and $\left\{\beta_{n}\right\}$ be the sequences of nonnegative real numbers and a sequence of real numbers, respectively, such that $\lim _{\sup } \operatorname{si\infty }_{n} \alpha_{n}<\infty$ and $\lim \sup _{n \rightarrow \infty} \beta_{n} \leq$ 0 . Then $\limsup _{n \rightarrow \infty} \alpha_{n} \beta_{n} \leq 0$. 


\section{Main results}

In this section, we will introduce and analyze a multistep Mann-type extragradient iterative algorithm for finding a solution of SHVI (1.10) (over the fixed point set of an infinite family of nonexpansive mappings and a strict pseudocontraction) with constraints of several problems: finitely many GMEPs, finitely many variational inclusions and VIP (1.1) in a real Hilbert space. This algorithm is based on Korpelevich's extragradient method, viscosity approximation method, hybrid steepest-descent method, Mann's iteration method and projection method. We prove the strong convergence of the proposed algorithm to a unique solution of SHVI (1.10) under suitable conditions.

We are now in a position to state and prove the main result in this paper.

Theorem 3.1 Let $C$ be a nonempty closed convex subset of a real Hilbert space $H$. Let $M, N$ be two positive integers. Let $\Theta_{k}$ be a bifunction from $C \times C$ to $\mathbf{R}$ satisfying (A1)(A4) and $\varphi_{k}: C \rightarrow \mathbf{R} \cup\{+\infty\}$ be a proper lower semicontinuous and convex function with restriction (B1) or (B2), where $k \in\{1,2, \ldots, M\}$. Let $R_{i}: C \rightarrow 2^{H}$ be a maximal monotone mapping and let $A_{k}: H \rightarrow H$ and $B_{i}: C \rightarrow H$ be $\mu_{k}$-inverse-strongly monotone and $\eta_{i}$-inverse-strongly monotone, respectively, where $k \in\{1,2, \ldots, M\}, i \in\{1,2, \ldots, N\}$. Let $\left\{T_{n}\right\}_{n=1}^{\infty}$ be a sequence of nonexpansive self-mappings on $C$ and $\left\{\lambda_{n}\right\}_{n=1}^{\infty}$ be a sequence in $(0, b]$ for some $b \in(0,1)$. Let $T: C \rightarrow C$ be a $\xi$-strictly pseudocontractive mapping, $S: C \rightarrow C$ be a nonexpansive mapping and $V: C \rightarrow H$ be a $\rho$-contraction with coeffcient $\rho \in[0,1)$. Let $A: C \rightarrow H$ be a $\frac{1}{L}$-inverse-strongly monotone mapping, and $F: C \rightarrow H$ be $\kappa$-Lipschitzian and $\eta$-strongly monotone with positive constants $\kappa, \eta>0$ such that $0<\mu<\frac{2 \eta}{\kappa^{2}}$ and $0<\gamma \leq \tau$ where $\tau=1-\sqrt{1-\mu\left(2 \eta-\mu \kappa^{2}\right)}$. Assume that SHVI (1.10) over $\Omega:=\bigcap_{n=1}^{\infty} \operatorname{Fix}\left(T_{n}\right) \cap \bigcap_{k=1}^{M} \operatorname{GMEP}\left(\Theta_{k}, \varphi_{k}, A_{k}\right) \cap \bigcap_{i=1}^{N} \mathrm{I}\left(B_{i}, R_{i}\right) \cap \operatorname{VI}(C, A) \cap \operatorname{Fix}(T)$ has a solution. Let $\left\{\alpha_{n}\right\} \subset[0, \infty),\left\{v_{n}\right\} \subset\left(0, \frac{1}{L}\right),\left\{\epsilon_{n}\right\},\left\{\delta_{n}\right\},\left\{\beta_{n}\right\},\left\{\gamma_{n}\right\},\left\{\sigma_{n}\right\} \subset(0,1)$, and $\left\{\lambda_{i, n}\right\} \subset$ $\left[a_{i}, b_{i}\right] \subset\left(0,2 \eta_{i}\right),\left\{r_{k, n}\right\} \subset\left[c_{k}, d_{k}\right] \subset\left(0,2 \mu_{k}\right)$ where $i \in\{1,2, \ldots, N\}$ and $k \in\{1,2, \ldots, M\}$. For arbitrarily given $x_{1} \in C$, let $\left\{x_{n}\right\}$ be a sequence generated by

$$
\left\{\begin{array}{l}
u_{n}=T_{r_{M, n}}^{\left(\Theta_{M}, \varphi_{M}\right)}\left(I-r_{M, n} A_{M}\right) T_{r_{M-1, n}}^{\left(\Theta_{M-1}, \varphi_{M-1}\right)}\left(I-r_{M-1, n} A_{M-1}\right) \cdots T_{r_{1, n}}^{\left(\Theta_{1}, \varphi_{1}\right)}\left(I-r_{1, n} A_{1}\right) x_{n}, \\
v_{n}=J_{R_{N}, \lambda_{N, n}}\left(I-\lambda_{N, n} B_{N}\right) J_{R_{N-1}, \lambda_{N-1, n}}\left(I-\lambda_{N-1, n} B_{N-1}\right) \cdots J_{R_{1}, \lambda_{1, n}}\left(I-\lambda_{1, n} B_{1}\right) u_{n}, \\
y_{n}=P_{C}\left(v_{n}-v_{n} A_{n} v_{n}\right) \\
z_{n}=\beta_{n} W_{n} x_{n}+\gamma_{n} P_{C}\left(v_{n}-v_{n} A_{n} y_{n}\right)+\sigma_{n} T P_{C}\left(v_{n}-v_{n} A_{n} y_{n}\right), \\
x_{n+1}=P_{C}\left[\epsilon_{n} \gamma\left(\delta_{n} V x_{n}+\left(1-\delta_{n}\right) S x_{n}\right)+\left(I-\epsilon_{n} \mu F\right) z_{n}\right], \quad \forall n \geq 1,
\end{array}\right.
$$

where $A_{n}=\alpha_{n} I+A$ for all $n \geq 1$. Suppose that

(C1) $\sum_{n=1}^{\infty} \alpha_{n}<\infty$;

(C2) $0<\liminf _{n \rightarrow \infty} v_{n} \leq \limsup _{n \rightarrow \infty} v_{n}<\frac{1}{L}$;

(C3) $\beta_{n}+\gamma_{n}+\sigma_{n}=1$ and $\left(\gamma_{n}+\sigma_{n}\right) \xi \leq \gamma_{n}$ for all $n \geq 1$;

(C4) $0<\liminf _{n \rightarrow \infty} \beta_{n} \leq \limsup _{n \rightarrow \infty} \beta_{n}<1$ and $\liminf _{n \rightarrow \infty} \sigma_{n}>0$;

(C5) $0<\liminf _{n \rightarrow \infty} \delta_{n} \leq \limsup _{n \rightarrow \infty} \delta_{n}<1$;

(C6) $\lim _{n \rightarrow \infty} \epsilon_{n}=0$ and $\sum_{n=1}^{\infty} \epsilon_{n}=\infty$.

If $\left\{S x_{n}\right\}$ is bounded, then $\left\{x_{n}\right\}$ converges strongly to a unique solution of SHVI(1.10) provided $\lim _{n \rightarrow \infty}\left\|x_{n}-x_{n+1}\right\|=0$.

Proof For $n \geq 1$, put

$$
\Delta_{n}^{k}=T_{r_{k, n}}^{\left(\Theta_{k}, \varphi_{k}\right)}\left(I-r_{k, n} A_{k}\right) T_{r_{k-1, n}}^{\left(\Theta_{k-1}, \varphi_{k-1}\right)}\left(I-r_{k-1, n} A_{k-1}\right) \cdots T_{r_{1, n}}^{\left(\Theta_{1}, \varphi_{1}\right)}\left(I-r_{1, n} A_{1}\right) x_{n}
$$


for all $k \in\{1,2, \ldots, M\}$ and

$$
\Lambda_{n}^{i}=J_{R_{i}, \lambda_{i, n}}\left(I-\lambda_{i, n} B_{i}\right) J_{R_{i-1}, \lambda_{i-1, n}}\left(I-\lambda_{i-1, n} B_{i-1}\right) \cdots J_{R_{1}, \lambda_{1, n}}\left(I-\lambda_{1, n} B_{1}\right)
$$

for all $i \in\{1,2, \ldots, N\}, \Delta_{n}^{0}=I$ and $\Lambda_{n}^{0}=I$. Then we have $u_{n}=\Delta_{n}^{M} x_{n}$ and $v_{n}=\Lambda_{n}^{N} u_{n}$. In addition, in terms of conditions $(\mathrm{C} 1),(\mathrm{C} 2)$, and $(\mathrm{C} 4)$, without loss of generality, we may assume that $\left\{\beta_{n}\right\} \subset[c, d]$ for some $c, d \in(0,1),\left\{v_{n}\right\} \subset[\hat{a}, \hat{b}]$ for some $\hat{a}, \hat{b} \in\left(0, \frac{1}{L}\right)$, and $v_{n}\left(\alpha_{n}+L\right) \leq 1$ for all $n \geq 1$.

One can readily see that $P_{C}\left(I-v_{n} A_{n}\right)$ are nonexpansive for all $n \geq 1$; see [7] (also [47]). Next, we divide the remainder of the proof into several steps.

Step 1. $\left\{x_{n}\right\}$ is bounded.

Take a fixed $p \in \Omega$ arbitrarily. Utilizing (2.2) and Proposition 2.6(ii) we have

$$
\begin{aligned}
\left\|u_{n}-p\right\|= & \left\|T_{r_{M, n}}^{\left(\Theta_{M}, \varphi_{M}\right)}\left(I-r_{M, n} B_{M}\right) \Delta_{n}^{M-1} x_{n}-T_{r_{M, n}}^{\left(\Theta_{M}, \varphi_{M}\right)}\left(I-r_{M, n} B_{M}\right) \Delta_{n}^{M-1} p\right\| \\
\leq & \left\|\left(I-r_{M, n} B_{M}\right) \Delta_{n}^{M-1} x_{n}-\left(I-r_{M, n} B_{M}\right) \Delta_{n}^{M-1} p\right\| \\
\leq & \left\|\Delta_{n}^{M-1} x_{n}-\Delta_{n}^{M-1} p\right\| \\
& \cdots \\
\leq & \left\|\Delta_{n}^{0} x_{n}-\Delta_{n}^{0} p\right\| \\
= & \left\|x_{n}-p\right\| .
\end{aligned}
$$

Utilizing (2.2) and Lemma 2.8 we have

$$
\begin{aligned}
\left\|v_{n}-p\right\|= & \left\|J_{R_{N}, \lambda_{N, n}}\left(I-\lambda_{N, n} A_{N}\right) \Lambda_{n}^{N-1} u_{n}-J_{R_{N}, \lambda_{N, n}}\left(I-\lambda_{N, n} A_{N}\right) \Lambda_{n}^{N-1} p\right\| \\
\leq & \left\|\left(I-\lambda_{N, n} A_{N}\right) \Lambda_{n}^{N-1} u_{n}-\left(I-\lambda_{N, n} A_{N}\right) \Lambda_{n}^{N-1} p\right\| \\
\leq & \left\|\Lambda_{n}^{N-1} u_{n}-\Lambda_{n}^{N-1} p\right\| \\
& \cdots \\
\leq & \left\|\Lambda_{n}^{0} u_{n}-\Lambda_{n}^{0} p\right\| \\
= & \left\|u_{n}-p\right\|
\end{aligned}
$$

which together with the last inequality, implies that

$$
\left\|v_{n}-p\right\| \leq\left\|x_{n}-p\right\|
$$

Note that $W_{n} p=p$ for all $n \geq 1$ and $P_{C}(I-v A) p=p$ for $v \in\left(0, \frac{2}{L}\right)$. Hence, from (3.1) and (3.4), it follows that

$$
\begin{aligned}
\left\|y_{n}-p\right\| & =\left\|P_{C}\left(I-v_{n} A_{n}\right) v_{n}-P_{C}\left(I-v_{n} A\right) p\right\| \\
& \leq\left\|P_{C}\left(I-v_{n} A_{n}\right) v_{n}-P_{C}\left(I-v_{n} A_{n}\right) p\right\|+\left\|P_{C}\left(I-v_{n} A_{n}\right) p-P_{C}\left(I-v_{n} A\right) p\right\| \\
& \leq\left\|v_{n}-p\right\|+\left\|\left(I-v_{n} A_{n}\right) p-\left(I-v_{n} A\right) p\right\| \\
& \leq\left\|x_{n}-p\right\|+v_{n} \alpha_{n}\|p\| .
\end{aligned}
$$


Put $t_{n}=P_{C}\left(v_{n}-v_{n} A_{n} y_{n}\right)$ for each $n \geq 1$. Then, by Proposition 2.1(ii), we have

$$
\begin{aligned}
\left\|t_{n}-p\right\|^{2} \leq & \left\|v_{n}-v_{n} A_{n} y_{n}-p\right\|^{2}-\left\|v_{n}-v_{n} A_{n} y_{n}-t_{n}\right\|^{2} \\
= & \left\|v_{n}-p\right\|^{2}-\left\|v_{n}-t_{n}\right\|^{2}+2 v_{n}\left\langle A_{n} y_{n}, p-t_{n}\right\rangle \\
= & \left\|v_{n}-p\right\|^{2}-\left\|v_{n}-t_{n}\right\|^{2}+2 v_{n}\left(\left\langle A_{n} y_{n}-A_{n} p, p-y_{n}\right\rangle\right. \\
& \left.+\left\langle A_{n} p, p-y_{n}\right\rangle+\left\langle A_{n} y_{n}, y_{n}-t_{n}\right\rangle\right) \\
\leq & \left\|v_{n}-p\right\|^{2}-\left\|v_{n}-t_{n}\right\|^{2}+2 v_{n}\left(\left\langle A_{n} p, p-y_{n}\right\rangle+\left\langle A_{n} y_{n}, y_{n}-t_{n}\right\rangle\right) \\
= & \left\|v_{n}-p\right\|^{2}-\left\|v_{n}-t_{n}\right\|^{2}+2 v_{n}\left[\left\langle\left(\alpha_{n} I+A\right) p, p-y_{n}\right\rangle+\left\langle A_{n} y_{n}, y_{n}-t_{n}\right\rangle\right] \\
\leq & \left\|v_{n}-p\right\|^{2}-\left\|v_{n}-t_{n}\right\|^{2}+2 v_{n}\left[\alpha_{n}\left\langle p, p-y_{n}\right\rangle+\left\langle A_{n} y_{n}, y_{n}-t_{n}\right\rangle\right] \\
= & \left\|v_{n}-p\right\|^{2}-\left\|v_{n}-y_{n}\right\|^{2}-2\left\langle v_{n}-y_{n}, y_{n}-t_{n}\right\rangle-\left\|y_{n}-t_{n}\right\|^{2} \\
& +2 v_{n}\left[\alpha_{n}\left\langle p, p-y_{n}\right\rangle+\left\langle A_{n} y_{n}, y_{n}-t_{n}\right\rangle\right] \\
= & \left\|v_{n}-p\right\|^{2}-\left\|v_{n}-y_{n}\right\|^{2}-\left\|y_{n}-t_{n}\right\|^{2} \\
& +2\left\langle v_{n}-v_{n} A_{n} y_{n}-y_{n}, t_{n}-y_{n}\right\rangle+2 v_{n} \alpha_{n}\left\langle p, p-y_{n}\right\rangle .
\end{aligned}
$$

Further, by Proposition 2.1(i), we have

$$
\begin{aligned}
\left\langle v_{n}-v_{n} A_{n} y_{n}-y_{n}, t_{n}-y_{n}\right\rangle & =\left\langle v_{n}-v_{n} A_{n} v_{n}-y_{n}, t_{n}-y_{n}\right\rangle+\left\langle v_{n} A_{n} v_{n}-v_{n} A_{n} y_{n}, t_{n}-y_{n}\right\rangle \\
& \leq\left\langle v_{n} A_{n} v_{n}-v_{n} A_{n} y_{n}, t_{n}-y_{n}\right\rangle \\
& \leq v_{n}\left\|A_{n} v_{n}-A_{n} y_{n}\right\|\left\|t_{n}-y_{n}\right\| \\
& \leq v_{n}\left(\alpha_{n}+L\right)\left\|v_{n}-y_{n}\right\|\left\|t_{n}-y_{n}\right\| .
\end{aligned}
$$

So, we obtain from (3.6)

$$
\begin{aligned}
\left\|t_{n}-p\right\|^{2} \leq & \left\|v_{n}-p\right\|^{2}-\left\|v_{n}-y_{n}\right\|^{2}-\left\|y_{n}-t_{n}\right\|^{2}+2\left\langle v_{n}-v_{n} A_{n} y_{n}-y_{n}, t_{n}-y_{n}\right\rangle \\
& +2 v_{n} \alpha_{n}\left\langle p, p-y_{n}\right\rangle \\
\leq & \left\|v_{n}-p\right\|^{2}-\left\|v_{n}-y_{n}\right\|^{2}-\left\|y_{n}-t_{n}\right\|^{2}+2 v_{n}\left(\alpha_{n}+L\right)\left\|v_{n}-y_{n}\right\|\left\|t_{n}-y_{n}\right\| \\
& +2 v_{n} \alpha_{n}\|p\|\left\|p-y_{n}\right\| \\
\leq & \left\|v_{n}-p\right\|^{2}-\left\|v_{n}-y_{n}\right\|^{2}-\left\|y_{n}-t_{n}\right\|^{2}+v_{n}^{2}\left(\alpha_{n}+L\right)^{2}\left\|v_{n}-y_{n}\right\|^{2}+\left\|t_{n}-y_{n}\right\|^{2} \\
& +2 v_{n} \alpha_{n}\|p\|\left\|p-y_{n}\right\| \\
= & \left\|v_{n}-p\right\|^{2}+2 v_{n} \alpha_{n}\|p\|\left\|p-y_{n}\right\|+\left(v_{n}^{2}\left(\alpha_{n}+L\right)^{2}-1\right)\left\|v_{n}-y_{n}\right\|^{2} \\
\leq & \left\|x_{n}-p\right\|^{2}+2 v_{n} \alpha_{n}\|p\|\left\|p-y_{n}\right\|+\left(v_{n}^{2}\left(\alpha_{n}+L\right)^{2}-1\right)\left\|v_{n}-y_{n}\right\|^{2} \\
\leq & \left\|x_{n}-p\right\|^{2}+2 v_{n} \alpha_{n}\|p\|\left\|p-y_{n}\right\| .
\end{aligned}
$$

Since $\left(\gamma_{n}+\sigma_{n}\right) \xi \leq \gamma_{n}$ for all $n \geq 1$, utilizing Proposition 2.5 and Lemma 2.1(b), from (3.5) and (3.8), we conclude that

$$
\begin{aligned}
\left\|z_{n}-p\right\|^{2} & =\left\|\beta_{n} W_{n} x_{n}+\gamma_{n} t_{n}+\sigma_{n} T t_{n}-p\right\|^{2} \\
& =\left\|\beta_{n}\left(W_{n} x_{n}-p\right)+\left(\gamma_{n}+\sigma_{n}\right) \frac{1}{\gamma_{n}+\sigma_{n}}\left[\gamma_{n}\left(t_{n}-p\right)+\sigma_{n}\left(T t_{n}-p\right)\right]\right\|^{2}
\end{aligned}
$$




$$
\begin{aligned}
= & \beta_{n}\left\|W_{n} x_{n}-p\right\|^{2}+\left(\gamma_{n}+\sigma_{n}\right)\left\|\frac{1}{\gamma_{n}+\sigma_{n}}\left[\gamma_{n}\left(t_{n}-p\right)+\sigma_{n}\left(T t_{n}-p\right)\right]\right\|^{2} \\
& -\beta_{n}\left(\gamma_{n}+\sigma_{n}\right)\left\|\frac{1}{\gamma_{n}+\sigma_{n}}\left[\gamma_{n}\left(t_{n}-W_{n} x_{n}\right)+\sigma_{n}\left(T t_{n}-W_{n} x_{n}\right)\right]\right\|^{2} \\
\leq & \beta_{n}\left\|x_{n}-p\right\|^{2}+\left(1-\beta_{n}\right)\left\|t_{n}-p\right\|^{2}-\frac{\beta_{n}}{1-\beta_{n}}\left\|z_{n}-W_{n} x_{n}\right\|^{2} \\
\leq & \beta_{n}\left\|x_{n}-p\right\|^{2}+\left(1-\beta_{n}\right)\left[\left\|x_{n}-p\right\|^{2}+2 v_{n} \alpha_{n}\|p\|\left\|p-y_{n}\right\|\right. \\
& \left.+\left(v_{n}^{2}\left(\alpha_{n}+L\right)^{2}-1\right)\left\|v_{n}-y_{n}\right\|^{2}\right]-\frac{\beta_{n}}{1-\beta_{n}}\left\|z_{n}-W_{n} x_{n}\right\|^{2} \\
\leq & \left\|x_{n}-p\right\|^{2}+2 v_{n} \alpha_{n}\|p\|\left\|p-y_{n}\right\|+\left(1-\beta_{n}\right)\left(v_{n}^{2}\left(\alpha_{n}+L\right)^{2}-1\right)\left\|v_{n}-y_{n}\right\|^{2} \\
& -\frac{\beta_{n}}{1-\beta_{n}}\left\|z_{n}-W_{n} x_{n}\right\|^{2} \\
\leq & \left\|x_{n}-p\right\|^{2}+2 v_{n} \alpha_{n}\|p\|\left(\left\|x_{n}-p\right\|+v_{n} \alpha_{n}\|p\|\right) \\
& +\left(1-\beta_{n}\right)\left(v_{n}^{2}\left(\alpha_{n}+L\right)^{2}-1\right)\left\|v_{n}-y_{n}\right\|^{2}-\frac{\beta_{n}}{1-\beta_{n}}\left\|z_{n}-W_{n} x_{n}\right\|^{2} \\
\leq & \left\|x_{n}-p\right\|^{2}+2\left\|x_{n}-p\right\|\left(\sqrt{2} v_{n} \alpha_{n}\|p\|\right)+\left(\sqrt{2} v_{n} \alpha_{n}\|p\|\right)^{2} \\
& +\left(1-\beta_{n}\right)\left(v_{n}^{2}\left(\alpha_{n}+L\right)^{2}-1\right)\left\|v_{n}-y_{n}\right\|^{2}-\frac{\beta_{n}}{1-\beta_{n}}\left\|z_{n}-W_{n} x_{n}\right\|^{2} \\
= & \left(\left\|x_{n}-p\right\|+\sqrt{2} v_{n} \alpha_{n}\|p\|\right)^{2}+\left(1-\beta_{n}\right)\left(v_{n}^{2}\left(\alpha_{n}+L\right)^{2}-1\right)\left\|v_{n}-y_{n}\right\|^{2} \\
& -\frac{\beta_{n}}{1-\beta_{n}}\left\|z_{n}-W_{n} x_{n}\right\|^{2} \\
\leq & \left(\left\|x_{n}-p\right\|+\sqrt{2} v_{n} \alpha_{n}\|p\|\right)^{2} .
\end{aligned}
$$

Noticing the boundedness of $\left\{S x_{n}\right\}$, we get $\sup _{n \geq 1}\left\|\gamma S x_{n}-\mu F p\right\| \leq \widetilde{M}$ for some $\tilde{M}>0$.

Moreover, utilizing Lemma 2.5 we have from (3.1)

$$
\begin{aligned}
\left\|x_{n+1}-p\right\|= & \left\|P_{C}\left[\epsilon_{n} \gamma\left(\delta_{n} V x_{n}+\left(1-\delta_{n}\right) S x_{n}\right)+\left(I-\epsilon_{n} \mu F\right) z_{n}\right]-P_{C} p\right\| \\
\leq & \left\|\epsilon_{n} \gamma\left(\delta_{n} V x_{n}+\left(1-\delta_{n}\right) S x_{n}\right)+\left(I-\epsilon_{n} \mu F\right) z_{n}-p\right\| \\
= & \left\|\epsilon_{n} \gamma\left(\delta_{n} V x_{n}+\left(1-\delta_{n}\right) S x_{n}\right)-\epsilon_{n} \mu F p+\left(I-\epsilon_{n} \mu F\right) z_{n}-\left(I-\epsilon_{n} \mu F\right) p\right\| \\
\leq & \left\|\epsilon_{n} \gamma\left(\delta_{n} V x_{n}+\left(1-\delta_{n}\right) S x_{n}\right)-\epsilon_{n} \mu F p\right\|+\left\|\left(I-\epsilon_{n} \mu F\right) z_{n}-\left(I-\epsilon_{n} \mu F\right) p\right\| \\
= & \epsilon_{n}\left\|\delta_{n}\left(\gamma V x_{n}-\mu F p\right)+\left(1-\delta_{n}\right)\left(\gamma S x_{n}-\mu F p\right)\right\| \\
& +\left\|\left(I-\epsilon_{n} \mu F\right) z_{n}-\left(I-\epsilon_{n} \mu F\right) p\right\| \\
\leq & \epsilon_{n}\left[\delta_{n}\left\|\gamma V x_{n}-\mu F p\right\|+\left(1-\delta_{n}\right)\left\|\gamma S x_{n}-\mu F p\right\|\right]+\left(1-\epsilon_{n} \tau\right)\left\|z_{n}-p\right\| \\
\leq & \epsilon_{n}\left[\delta_{n}\left(\left\|\gamma V x_{n}-\gamma V p\right\|+\|\gamma V p-\mu F p\|\right)+\left(1-\delta_{n}\right) \tilde{M}\right]+\left(1-\epsilon_{n} \tau\right)\left\|z_{n}-p\right\| \\
\leq & \epsilon_{n}\left[\delta_{n} \gamma \rho\left\|x_{n}-p\right\|+\delta_{n}\|\gamma V p-\mu F p\|+\left(1-\delta_{n}\right) \tilde{M}\right] \\
& +\left(1-\epsilon_{n} \tau\right)\left[\left\|x_{n}-p\right\|+\sqrt{2} v_{n} \alpha_{n}\|p\|\right] \\
\leq & \epsilon_{n}\left[\delta_{n} \gamma \rho\left\|x_{n}-p\right\|+\max \{\tilde{M},\|\gamma V p-\mu F p\|\}\right] \\
& +\left(1-\epsilon_{n} \tau\right)\left[\left\|x_{n}-p\right\|+\sqrt{2} v_{n} \alpha_{n}\|p\|\right] \\
\leq & \epsilon_{n} \gamma \rho\left\|x_{n}-p\right\|+\epsilon_{n} \max \{\tilde{M},\|\gamma V p-\mu F p\|\}
\end{aligned}
$$




$$
\begin{aligned}
& +\left(1-\epsilon_{n} \tau\right)\left\|x_{n}-p\right\|+\sqrt{2} v_{n} \alpha_{n}\|p\| \\
= & {\left[1-(\tau-\gamma \rho) \epsilon_{n}\right]\left\|x_{n}-p\right\|+\epsilon_{n} \max \{\tilde{M},\|\gamma V p-\mu F p\|\}+\sqrt{2} v_{n} \alpha_{n}\|p\| } \\
= & {\left[1-(\tau-\gamma \rho) \epsilon_{n}\right]\left\|x_{n}-p\right\|+(\tau-\gamma \rho) \epsilon_{n} \max \left\{\frac{\tilde{M}}{\tau-\gamma \rho}, \frac{\|\gamma V p-\mu F p\|}{\tau-\gamma \rho}\right\} } \\
& +\sqrt{2} v_{n} \alpha_{n}\|p\| \\
\leq & \max \left\{\left\|x_{n}-p\right\|, \frac{\tilde{M}}{\tau-\gamma \rho}, \frac{\|\gamma V p-\mu F p\|}{\tau-\gamma \rho}\right\}+\sqrt{2} v_{n} \alpha_{n}\|p\| .
\end{aligned}
$$

By induction, we can derive

$$
\left\|x_{n+1}-p\right\| \leq \max \left\{\left\|x_{1}-p\right\|, \frac{\tilde{M}}{\tau-\gamma \rho}, \frac{\|\gamma V p-\mu F p\|}{\tau-\gamma \rho}\right\}+\sum_{j=1}^{n} \sqrt{2} v_{j} \alpha_{j}\|p\|, \quad \forall n \geq 1 .
$$

Consequently, $\left\{x_{n}\right\}$ is bounded (due to $\left.\sum_{n=1}^{\infty} \alpha_{n}<\infty\right)$ and so are the sequences $\left\{u_{n}\right\},\left\{v_{n}\right\}$, $\left\{y_{n}\right\},\left\{z_{n}\right\},\left\{A v_{n}\right\}$ and $\left\{A y_{n}\right\}$.

Step 2. $\left\|x_{n}-u_{n}\right\| \rightarrow 0,\left\|x_{n}-v_{n}\right\| \rightarrow 0,\left\|x_{n}-y_{n}\right\| \rightarrow 0,\left\|x_{n}-t_{n}\right\| \rightarrow 0,\left\|x_{n}-W x_{n}\right\| \rightarrow 0$ and $\left\|t_{n}-T t_{n}\right\| \rightarrow 0$ as $n \rightarrow \infty$.

From (3.1) and (3.9), it follows that

$$
\begin{aligned}
\left\|x_{n+1}-p\right\|^{2} \leq & \left\|\epsilon_{n} \gamma\left(\delta_{n} V x_{n}+\left(1-\delta_{n}\right) S x_{n}\right)+\left(I-\epsilon_{n} \mu F\right) z_{n}-p\right\|^{2} \\
= & \left\|\epsilon_{n} \gamma\left(\delta_{n} V x_{n}+\left(1-\delta_{n}\right) S x_{n}\right)-\epsilon_{n} \mu F p+\left(I-\epsilon_{n} \mu F\right) z_{n}-\left(I-\epsilon_{n} \mu F\right) p\right\|^{2} \\
\leq & \left\{\left\|\epsilon_{n} \gamma\left(\delta_{n} V x_{n}+\left(1-\delta_{n}\right) S x_{n}\right)-\epsilon_{n} \mu F p\right\|+\left\|\left(I-\epsilon_{n} \mu F\right) z_{n}-\left(I-\epsilon_{n} \mu F\right) p\right\|\right\}^{2} \\
\leq & \left\{\epsilon_{n}\left\|\delta_{n}\left(\gamma V x_{n}-\mu F p\right)+\left(1-\delta_{n}\right)\left(\gamma S x_{n}-\mu F p\right)\right\|+\left(1-\epsilon_{n} \tau\right)\left\|z_{n}-p\right\|\right\}^{2} \\
\leq & \epsilon_{n} \frac{1}{\tau}\left[\delta_{n}\left\|\gamma V x_{n}-\mu F p\right\|+\left(1-\delta_{n}\right)\left\|\gamma S x_{n}-\mu F p\right\|\right]^{2}+\left(1-\epsilon_{n} \tau\right)\left\|z_{n}-p\right\|^{2} \\
\leq & \epsilon_{n} \frac{1}{\tau}\left[\left\|\gamma V x_{n}-\mu F p\right\|+\left\|\gamma S x_{n}-\mu F p\right\|\right]^{2}+\left\|z_{n}-p\right\|^{2} \\
\leq & \epsilon_{n} \frac{1}{\tau}\left[\left\|\gamma V x_{n}-\mu F p\right\|+\left\|\gamma S x_{n}-\mu F p\right\|\right]^{2}+\left(\left\|x_{n}-p\right\|+\sqrt{2} v_{n} \alpha_{n}\|p\|\right)^{2} \\
& +\left(1-\beta_{n}\right)\left(v_{n}^{2}\left(\alpha_{n}+L\right)^{2}-1\right)\left\|v_{n}-y_{n}\right\|^{2}-\frac{\beta_{n}}{1-\beta_{n}}\left\|z_{n}-W_{n} x_{n}\right\|^{2} \\
\leq & \left(\left\|x_{n}-p\right\|+\sqrt{2} v_{n} \alpha_{n}\|p\|\right)^{2}+\epsilon_{n} \tilde{M}_{1}+\left(1-\beta_{n}\right)\left(v_{n}^{2}\left(\alpha_{n}+L\right)^{2}-1\right)\left\|v_{n}-y_{n}\right\|^{2} \\
& -\frac{\beta_{n}}{1-\beta_{n}}\left\|z_{n}-W_{n} x_{n}\right\|^{2},
\end{aligned}
$$

where $\widetilde{M}_{1}=\sup _{n \geq 1}\left\{\frac{1}{\tau}\left[\left\|\gamma V x_{n}-\mu F p\right\|+\left\|\gamma S x_{n}-\mu F p\right\|\right]^{2}\right\}$. This together with $\left\{v_{n}\right\} \subset[\hat{a}, \hat{b}] \subset$ $\left(0, \frac{1}{L}\right)$ and $\left\{\beta_{n}\right\} \subset[c, d] \subset(0,1)$ implies that

$$
\begin{aligned}
& (1-d)\left(1-\hat{b}^{2}\left(\alpha_{n}+L\right)^{2}\right)\left\|v_{n}-y_{n}\right\|^{2}+\frac{c}{1-c}\left\|z_{n}-W_{n} x_{n}\right\|^{2} \\
& \quad \leq\left(1-\beta_{n}\right)\left(1-v_{n}^{2}\left(\alpha_{n}+L\right)^{2}\right)\left\|v_{n}-y_{n}\right\|^{2}+\frac{\beta_{n}}{1-\beta_{n}}\left\|z_{n}-W_{n} x_{n}\right\|^{2} \\
& \quad \leq\left(\left\|x_{n}-p\right\|+\sqrt{2} v_{n} \alpha_{n}\|p\|\right)^{2}-\left\|x_{n+1}-p\right\|^{2}+\epsilon_{n} \widetilde{M}_{1} \\
& \quad=\left[\left(\left\|x_{n}-p\right\|+\sqrt{2} v_{n} \alpha_{n}\|p\|\right)-\left\|x_{n+1}-p\right\|\right]
\end{aligned}
$$




$$
\begin{aligned}
& \times\left[\left(\left\|x_{n}-p\right\|+\sqrt{2} v_{n} \alpha_{n}\|p\|\right)+\left\|x_{n+1}-p\right\|\right]+\epsilon_{n} \tilde{M}_{1} \\
\leq & {\left[\left\|x_{n+1}-x_{n}\right\|+\sqrt{2} v_{n} \alpha_{n}\|p\|\right]\left[\left\|x_{n}-p\right\|+\left\|x_{n+1}-p\right\|+\sqrt{2} v_{n} \alpha_{n}\|p\|\right]+\epsilon_{n} \widetilde{M}_{1} } \\
\leq & {\left[\left\|x_{n+1}-x_{n}\right\|+\sqrt{2} \hat{b} \alpha_{n}\|p\|\right]\left[\left\|x_{n}-p\right\|+\left\|x_{n+1}-p\right\|+\sqrt{2} \hat{b} \alpha_{n}\|p\|\right]+\epsilon_{n} \widetilde{M}_{1} . }
\end{aligned}
$$

Note that $\lim _{n \rightarrow \infty} \alpha_{n}=\lim _{n \rightarrow \infty} \epsilon_{n}=0$. Hence, taking into account the boundedness of $\left\{x_{n}\right\}$ and $\lim _{n \rightarrow \infty}\left\|x_{n+1}-x_{n}\right\|=0$, we deduce from (3.12) that

$$
\lim _{n \rightarrow \infty}\left\|v_{n}-y_{n}\right\|=\lim _{n \rightarrow \infty}\left\|z_{n}-W_{n} x_{n}\right\|=0
$$

Furthermore, for simplicity, we write $w_{n}=\epsilon_{n} \gamma\left(\delta_{n} V x_{n}+\left(1-\delta_{n}\right) S x_{n}\right)+\left(I-\epsilon_{n} \mu F\right) z_{n}$ for all $n \geq 1$. Then we have

$$
\begin{aligned}
x_{n+1}-x_{n} & =P_{C} w_{n}-w_{n}+\epsilon_{n} \gamma\left(\delta_{n} V x_{n}+\left(1-\delta_{n}\right) S x_{n}\right)+\left(I-\epsilon_{n} \mu F\right) z_{n}-x_{n} \\
& =P_{C} w_{n}-w_{n}+\epsilon_{n}\left[\gamma\left(\delta_{n} V x_{n}+\left(1-\delta_{n}\right) S x_{n}\right)-\mu F z_{n}\right]+z_{n}-x_{n},
\end{aligned}
$$

which immediately yields

$$
z_{n}-x_{n}=x_{n+1}-x_{n}-\epsilon_{n}\left[\gamma\left(\delta_{n} V x_{n}+\left(1-\delta_{n}\right) S x_{n}\right)-\mu F z_{n}\right]-\left(P_{C} w_{n}-w_{n}\right) .
$$

So, utilizing Proposition 2.1(i) we get

$$
\begin{aligned}
\left\|z_{n}-x_{n}\right\|^{2}= & \left\|x_{n+1}-x_{n}-\epsilon_{n}\left[\gamma\left(\delta_{n} V x_{n}+\left(1-\delta_{n}\right) S x_{n}\right)-\mu F z_{n}\right]-\left(P_{C} w_{n}-w_{n}\right)\right\|^{2} \\
\leq & \left\|x_{n+1}-x_{n}-\epsilon_{n}\left[\gamma\left(\delta_{n} V x_{n}+\left(1-\delta_{n}\right) S x_{n}\right)-\mu F z_{n}\right]\right\|^{2} \\
& -2\left\langle P_{C} w_{n}-w_{n}, z_{n}-x_{n}\right\rangle \\
= & \left\|x_{n+1}-x_{n}-\epsilon_{n}\left[\gamma\left(\delta_{n} V x_{n}+\left(1-\delta_{n}\right) S x_{n}\right)-\mu F z_{n}\right]\right\|^{2} \\
& -2\left(\left\langle P_{C} w_{n}-w_{n}, z_{n}-P_{C} w_{n}\right\rangle+\left\langle P_{C} w_{n}-w_{n}, P_{C} w_{n}-x_{n}\right\rangle\right) \\
\leq & {\left[\left\|x_{n+1}-x_{n}\right\|+\epsilon_{n}\left\|\gamma\left(\delta_{n} V x_{n}+\left(1-\delta_{n}\right) S x_{n}\right)-\mu F z_{n}\right\|\right]^{2} } \\
& +2\left(\left\langle P_{C} w_{n}-w_{n}, P_{C} w_{n}-z_{n}\right\rangle+\left\|P_{C} w_{n}-w_{n}\right\|\left\|P_{C} w_{n}-x_{n}\right\|\right) \\
\leq & 2\left\|x_{n+1}-x_{n}\right\|^{2}+2 \epsilon_{n}^{2}\left\|\gamma\left(\delta_{n} V x_{n}+\left(1-\delta_{n}\right) S x_{n}\right)-\mu F z_{n}\right\|^{2} \\
& +2\left\|x_{n+1}-w_{n}\right\|\left\|x_{n+1}-x_{n}\right\| .
\end{aligned}
$$

Since $\left\|x_{n+1}-x_{n}\right\| \rightarrow 0$ and $\epsilon_{n} \rightarrow 0$ (due to (C6)), we know from the boundedness of $\left\{w_{n}\right\}$, $\left\{x_{n}\right\}$, and $\left\{z_{n}\right\}$ that

$$
\lim _{n \rightarrow \infty}\left\|z_{n}-x_{n}\right\|=0
$$

Taking into account that $\left\|W_{n} x_{n}-x_{n}\right\| \leq\left\|W_{n} x_{n}-z_{n}\right\|+\left\|z_{n}-x_{n}\right\|$, we obtain from (3.13) and (3.14)

$$
\lim _{n \rightarrow \infty}\left\|x_{n}-W_{n} x_{n}\right\|=0
$$


Next let us show that $\lim _{n \rightarrow \infty}\left\|x_{n}-y_{n}\right\|=0$. As a matter of fact, from (3.4) and (3.8) it follows that

$$
\begin{aligned}
\left\|t_{n}-p\right\|^{2} \leq & \left\|v_{n}-p\right\|^{2}-\left\|v_{n}-y_{n}\right\|^{2}-\left\|y_{n}-t_{n}\right\|^{2}+2 v_{n}\left(\alpha_{n}+L\right)\left\|v_{n}-y_{n}\right\|\left\|t_{n}-y_{n}\right\| \\
& +2 v_{n} \alpha_{n}\|p\|\left\|p-y_{n}\right\| \\
\leq & \left\|v_{n}-p\right\|^{2}-\left\|v_{n}-y_{n}\right\|^{2}-\left\|y_{n}-t_{n}\right\|^{2}+v_{n}^{2}\left(\alpha_{n}+L\right)^{2}\left\|t_{n}-y_{n}\right\|^{2}+\left\|v_{n}-y_{n}\right\|^{2} \\
& +2 v_{n} \alpha_{n}\|p\|\left\|p-y_{n}\right\| \\
= & \left\|v_{n}-p\right\|^{2}+2 v_{n} \alpha_{n}\|p\|\left\|p-y_{n}\right\|+\left(v_{n}^{2}\left(\alpha_{n}+L\right)^{2}-1\right)\left\|t_{n}-y_{n}\right\|^{2} \\
\leq & \left\|x_{n}-p\right\|^{2}+2 v_{n} \alpha_{n}\|p\|\left\|p-y_{n}\right\|+\left(v_{n}^{2}\left(\alpha_{n}+L\right)^{2}-1\right)\left\|t_{n}-y_{n}\right\|^{2} .
\end{aligned}
$$

Utilizing Lemma 2.1(b), from (3.9) and (3.16), we obtain

$$
\begin{aligned}
\left\|z_{n}-p\right\|^{2} \leq & \beta_{n}\left\|x_{n}-p\right\|^{2}+\left(1-\beta_{n}\right)\left\|t_{n}-p\right\|^{2}-\frac{\beta_{n}}{1-\beta_{n}}\left\|z_{n}-W_{n} x_{n}\right\|^{2} \\
\leq & \beta_{n}\left\|x_{n}-p\right\|^{2}+\left(1-\beta_{n}\right)\left\|t_{n}-p\right\|^{2} \\
\leq & \beta_{n}\left\|x_{n}-p\right\|^{2}+\left(1-\beta_{n}\right)\left[\left\|x_{n}-p\right\|^{2}+2 v_{n} \alpha_{n}\|p\|\left\|p-y_{n}\right\|\right. \\
& \left.\quad+\left(v_{n}^{2}\left(\alpha_{n}+L\right)^{2}-1\right)\left\|t_{n}-y_{n}\right\|^{2}\right] \\
\leq & \left\|x_{n}-p\right\|^{2}+2 v_{n} \alpha_{n}\|p\|\left\|p-y_{n}\right\|+\left(1-\beta_{n}\right)\left(v_{n}^{2}\left(\alpha_{n}+L\right)^{2}-1\right)\left\|t_{n}-y_{n}\right\|^{2},
\end{aligned}
$$

which immediately implies that

$$
\begin{aligned}
(1- & d)\left(1-\hat{b}^{2}\left(\alpha_{n}+L\right)^{2}\right)\left\|t_{n}-y_{n}\right\|^{2} \\
& \leq\left(1-\beta_{n}\right)\left(1-v_{n}^{2}\left(\alpha_{n}+L\right)^{2}\right)\left\|t_{n}-y_{n}\right\|^{2} \\
& \leq\left\|x_{n}-p\right\|^{2}-\left\|z_{n}-p\right\|^{2}+2 v_{n} \alpha_{n}\|p\|\left\|p-y_{n}\right\| \\
& \leq\left\|x_{n}-z_{n}\right\|\left(\left\|x_{n}-p\right\|+\left\|z_{n}-p\right\|\right)+2 \hat{b} \alpha_{n}\|p\|\left\|p-y_{n}\right\| .
\end{aligned}
$$

Since $\alpha_{n} \rightarrow 0,\left\|x_{n}-z_{n}\right\| \rightarrow 0$ (due to (3.14)) and $\left\{x_{n}\right\},\left\{y_{n}\right\},\left\{z_{n}\right\}$ are bounded, we get

$$
\lim _{n \rightarrow \infty}\left\|t_{n}-y_{n}\right\|=0
$$

In the meantime, from (3.8) and (3.9) it is not hard to find that

$$
\begin{aligned}
\left\|z_{n}-p\right\|^{2} \leq & \beta_{n}\left\|x_{n}-p\right\|^{2}+\left(1-\beta_{n}\right)\left\|t_{n}-p\right\|^{2} \\
\leq & \beta_{n}\left\|x_{n}-p\right\|^{2}+\left(1-\beta_{n}\right)\left[\left\|v_{n}-p\right\|^{2}+2 v_{n} \alpha_{n}\|p\|\left\|p-y_{n}\right\|\right. \\
& \left.\quad+\left(v_{n}^{2}\left(\alpha_{n}+L\right)^{2}-1\right)\left\|v_{n}-y_{n}\right\|^{2}\right] \\
\leq & \beta_{n}\left\|x_{n}-p\right\|^{2}+\left(1-\beta_{n}\right)\left\|v_{n}-p\right\|^{2}+2 v_{n} \alpha_{n}\|p\|\left\|p-y_{n}\right\| .
\end{aligned}
$$

Now, let us show that $\lim _{n \rightarrow \infty}\left\|x_{n}-u_{n}\right\|=\lim _{n \rightarrow \infty}\left\|x_{n}-v_{n}\right\|=0$. In fact, observe that

$$
\begin{aligned}
\left\|\Delta_{n}^{k} x_{n}-p\right\|^{2} & =\left\|T_{r_{k, n}}^{\left(\Theta_{k}, \varphi_{k}\right)}\left(I-r_{k, n} A_{k}\right) \Delta_{n}^{k-1} x_{n}-T_{r_{k, n}}^{\left(\Theta_{k}, \varphi_{k}\right)}\left(I-r_{k, n} A_{k}\right) p\right\|^{2} \\
& \leq\left\|\left(I-r_{k, n} A_{k}\right) \Delta_{n}^{k-1} x_{n}-\left(I-r_{k, n} A_{k}\right) p\right\|^{2}
\end{aligned}
$$




$$
\begin{aligned}
& \leq\left\|\Delta_{n}^{k-1} x_{n}-p\right\|^{2}+r_{k, n}\left(r_{k, n}-2 \mu_{k}\right)\left\|A_{k} \Delta_{n}^{k-1} x_{n}-A_{k} p\right\|^{2} \\
& \leq\left\|x_{n}-p\right\|^{2}+r_{k, n}\left(r_{k, n}-2 \mu_{k}\right)\left\|A_{k} \Delta_{n}^{k-1} x_{n}-A_{k} p\right\|^{2}
\end{aligned}
$$

and

$$
\begin{aligned}
\left\|\Lambda_{n}^{i} u_{n}-p\right\|^{2} & =\left\|J_{R_{i}, \lambda_{i, n}}\left(I-\lambda_{i, n} B_{i}\right) \Lambda_{n}^{i-1} u_{n}-J_{R_{i}, \lambda_{i, n}}\left(I-\lambda_{i, n} B_{i}\right) p\right\|^{2} \\
& \leq\left\|\left(I-\lambda_{i, n} B_{i}\right) \Lambda_{n}^{i-1} u_{n}-\left(I-\lambda_{i, n} B_{i}\right) p\right\|^{2} \\
& \leq\left\|\Lambda_{n}^{i-1} u_{n}-p\right\|^{2}+\lambda_{i, n}\left(\lambda_{i, n}-2 \eta_{i}\right)\left\|B_{i} \Lambda_{n}^{i-1} u_{n}-B_{i} p\right\|^{2} \\
& \leq\left\|u_{n}-p\right\|^{2}+\lambda_{i, n}\left(\lambda_{i, n}-2 \eta_{i}\right)\left\|B_{i} \Lambda_{n}^{i-1} u_{n}-B_{i} p\right\|^{2} \\
& \leq\left\|x_{n}-p\right\|^{2}+\lambda_{i, n}\left(\lambda_{i, n}-2 \eta_{i}\right)\left\|B_{i} \Lambda_{n}^{i-1} u_{n}-B_{i} p\right\|^{2}
\end{aligned}
$$

for $i \in\{1,2, \ldots, N\}$ and $k \in\{1,2, \ldots, M\}$. Combining (3.18), (3.19), and (3.20), we get

$$
\begin{aligned}
\left\|z_{n}-p\right\|^{2} \leq & \beta_{n}\left\|x_{n}-p\right\|^{2}+\left(1-\beta_{n}\right)\left\|v_{n}-p\right\|^{2}+2 v_{n} \alpha_{n}\|p\|\left\|p-y_{n}\right\| \\
\leq & \beta_{n}\left\|x_{n}-p\right\|^{2}+\left(1-\beta_{n}\right)\left\|\Lambda_{n}^{i} u_{n}-p\right\|^{2}+2 v_{n} \alpha_{n}\|p\|\left\|p-y_{n}\right\| \\
\leq & \beta_{n}\left\|x_{n}-p\right\|^{2}+\left(1-\beta_{n}\right)\left[\left\|u_{n}-p\right\|^{2}+\lambda_{i, n}\left(\lambda_{i, n}-2 \eta_{i}\right)\left\|B_{i} \Lambda_{n}^{i-1} u_{n}-B_{i} p\right\|^{2}\right] \\
& +2 v_{n} \alpha_{n}\|p\|\left\|p-y_{n}\right\| \\
\leq & \beta_{n}\left\|x_{n}-p\right\|^{2}+\left(1-\beta_{n}\right)\left[\left\|\Delta_{n}^{k} x_{n}-p\right\|^{2}+\lambda_{i, n}\left(\lambda_{i, n}-2 \eta_{i}\right)\left\|B_{i} \Lambda_{n}^{i-1} u_{n}-B_{i} p\right\|^{2}\right] \\
& +2 v_{n} \alpha_{n}\|p\|\left\|p-y_{n}\right\| \\
\leq & \beta_{n}\left\|x_{n}-p\right\|^{2}+\left(1-\beta_{n}\right)\left[\left\|x_{n}-p\right\|^{2}+r_{k, n}\left(r_{k, n}-2 \mu \mu_{k}\right)\left\|A_{k} \Delta_{n}^{k-1} x_{n}-A_{k} p\right\|^{2}\right. \\
& \left.+\lambda_{i, n}\left(\lambda_{i, n}-2 \eta_{i}\right)\left\|B_{i} \Lambda_{n}^{i-1} u_{n}-B_{i} p\right\|^{2}\right]+2 v_{n} \alpha_{n}\|p\|\left\|p-y_{n}\right\| \\
= & \left\|x_{n}-p\right\|^{2}+\left(1-\beta_{n}\right)\left[r_{k, n}\left(r_{k, n}-2 \mu_{k}\right)\left\|A_{k} \Delta_{n}^{k-1} x_{n}-A_{k} p\right\|^{2}\right. \\
& \left.+\lambda_{i, n}\left(\lambda_{i, n}-2 \eta_{i}\right)\left\|B_{i} \Lambda_{n}^{i-1} u_{n}-B_{i} p\right\|^{2}\right]+2 v_{n} \alpha_{n}\|p\|\left\|p-y_{n}\right\|,
\end{aligned}
$$

which hence implies that

$$
\begin{aligned}
& (1-d)\left[r_{k, n}\left(2 \mu_{k}-r_{k, n}\right)\left\|A_{k} \Delta_{n}^{k-1} x_{n}-A_{k} p\right\|^{2}+\lambda_{i, n}\left(2 \eta_{i}-\lambda_{i, n}\right)\left\|B_{i} \Lambda_{n}^{i-1} u_{n}-B_{i} p\right\|^{2}\right] \\
& \quad \leq\left(1-\beta_{n}\right)\left[r_{k, n}\left(2 \mu_{k}-r_{k, n}\right)\left\|A_{k} \Delta_{n}^{k-1} x_{n}-A_{k} p\right\|^{2}+\lambda_{i, n}\left(2 \eta_{i}-\lambda_{i, n}\right)\left\|B_{i} \Lambda_{n}^{i-1} u_{n}-B_{i} p\right\|^{2}\right] \\
& \quad \leq\left\|x_{n}-p\right\|^{2}-\left\|z_{n}-p\right\|^{2}+2 v_{n} \alpha_{n}\|p\|\left\|p-y_{n}\right\| \\
& \quad \leq\left\|x_{n}-z_{n}\right\|\left(\left\|x_{n}-p\right\|+\left\|z_{n}-p\right\|\right)+2 v_{n} \alpha_{n}\|p\|\left\|p-y_{n}\right\| .
\end{aligned}
$$

Since $\alpha_{n} \rightarrow 0,\left\{v_{n}\right\} \subset[\hat{a}, \hat{b}] \subset\left(0, \frac{1}{L}\right),\left\{\lambda_{i, n}\right\} \subset\left[a_{i}, b_{i}\right] \subset\left(0,2 \eta_{i}\right)$ and $\left\{r_{k, n}\right\} \subset\left[c_{k}, d_{k}\right] \subset\left(0,2 \mu_{k}\right)$ where $i \in\{1,2, \ldots, N\}$ and $k \in\{1,2, \ldots, M\}$, we deduce from (3.14) and the boundedness of $\left\{x_{n}\right\},\left\{y_{n}\right\},\left\{z_{n}\right\}$ that

$$
\lim _{n \rightarrow \infty}\left\|A_{k} \Delta_{n}^{k-1} x_{n}-A_{k} p\right\|=0 \quad \text { and } \quad \lim _{n \rightarrow \infty}\left\|B_{i} \Lambda_{n}^{i-1} u_{n}-B_{i} p\right\|=0
$$

where $i \in\{1,2, \ldots, N\}$ and $k \in\{1,2, \ldots, M\}$. 
Furthermore, by Proposition 2.6(ii) and Lemma 2.1(a) we have

$$
\begin{aligned}
\left\|\Delta_{n}^{k} x_{n}-p\right\|^{2}= & \left\|T_{r_{k, n}}^{\left(\Theta_{k}, \varphi_{k}\right)}\left(I-r_{k, n} A_{k}\right) \Delta_{n}^{k-1} x_{n}-T_{r_{k, n}}^{\left(\Theta_{k}, \varphi_{k}\right)}\left(I-r_{k, n} A_{k}\right) p\right\|^{2} \\
\leq & \left\langle\left(I-r_{k, n} A_{k}\right) \Delta_{n}^{k-1} x_{n}-\left(I-r_{k, n} A_{k}\right) p, \Delta_{n}^{k} x_{n}-p\right\rangle \\
= & \frac{1}{2}\left(\left\|\left(I-r_{k, n} A_{k}\right) \Delta_{n}^{k-1} x_{n}-\left(I-r_{k, n} A_{k}\right) p\right\|^{2}+\left\|\Delta_{n}^{k} x_{n}-p\right\|^{2}\right. \\
& \left.-\left\|\left(I-r_{k, n} A_{k}\right) \Delta_{n}^{k-1} x_{n}-\left(I-r_{k, n} A_{k}\right) p-\left(\Delta_{n}^{k} x_{n}-p\right)\right\|^{2}\right) \\
\leq & \frac{1}{2}\left(\left\|\Delta_{n}^{k-1} x_{n}-p\right\|^{2}+\left\|\Delta_{n}^{k} x_{n}-p\right\|^{2}\right. \\
& \left.-\left\|\Delta_{n}^{k-1} x_{n}-\Delta_{n}^{k} x_{n}-r_{k, n}\left(A_{k} \Delta_{n}^{k-1} x_{n}-A_{k} p\right)\right\|^{2}\right),
\end{aligned}
$$

which implies that

$$
\begin{aligned}
\left\|\Delta_{n}^{k} x_{n}-p\right\|^{2} \leq & \left\|\Delta_{n}^{k-1} x_{n}-p\right\|^{2}-\left\|\Delta_{n}^{k-1} x_{n}-\Delta_{n}^{k} x_{n}-r_{k, n}\left(A_{k} \Delta_{n}^{k-1} x_{n}-A_{k} p\right)\right\|^{2} \\
= & \left\|\Delta_{n}^{k-1} x_{n}-p\right\|^{2}-\left\|\Delta_{n}^{k-1} x_{n}-\Delta_{n}^{k} x_{n}\right\|^{2}-r_{k, n}^{2}\left\|A_{k} \Delta_{n}^{k-1} x_{n}-A_{k} p\right\|^{2} \\
& +2 r_{k, n}\left\langle\Delta_{n}^{k-1} x_{n}-\Delta_{n}^{k} x_{n}, A_{k} \Delta_{n}^{k-1} x_{n}-A_{k} p\right\rangle \\
\leq & \left\|\Delta_{n}^{k-1} x_{n}-p\right\|^{2}-\left\|\Delta_{n}^{k-1} x_{n}-\Delta_{n}^{k} x_{n}\right\|^{2} \\
& +2 r_{k, n}\left\|\Delta_{n}^{k-1} x_{n}-\Delta_{n}^{k} x_{n}\right\|\left\|A_{k} \Delta_{n}^{k-1} x_{n}-A_{k} p\right\| \\
\leq & \left\|x_{n}-p\right\|^{2}-\left\|\Delta_{n}^{k-1} x_{n}-\Delta_{n}^{k} x_{n}\right\|^{2} \\
& +2 r_{k, n}\left\|\Delta_{n}^{k-1} x_{n}-\Delta_{n}^{k} x_{n}\right\|\left\|A_{k} \Delta_{n}^{k-1} x_{n}-A_{k} p\right\| .
\end{aligned}
$$

By Lemma 2.1(a) and Lemma 2.8, we obtain

$$
\begin{aligned}
\left\|\Lambda_{n}^{i} u_{n}-p\right\|^{2}= & \left\|J_{R_{i}, \lambda_{i, n}}\left(I-\lambda_{i, n} B_{i}\right) \Lambda_{n}^{i-1} u_{n}-J_{R_{i}, \lambda_{i, n}}\left(I-\lambda_{i, n} B_{i}\right) p\right\|^{2} \\
\leq & \left\langle\left(I-\lambda_{i, n} B_{i}\right) \Lambda_{n}^{i-1} u_{n}-\left(I-\lambda_{i, n} B_{i}\right) p, \Lambda_{n}^{i} u_{n}-p\right\rangle \\
= & \frac{1}{2}\left(\left\|\left(I-\lambda_{i, n} B_{i}\right) \Lambda_{n}^{i-1} u_{n}-\left(I-\lambda_{i, n} B_{i}\right) p\right\|^{2}+\left\|\Lambda_{n}^{i} u_{n}-p\right\|^{2}\right. \\
& \left.-\left\|\left(I-\lambda_{i, n} B_{i}\right) \Lambda_{n}^{i-1} u_{n}-\left(I-\lambda_{i, n} B_{i}\right) p-\left(\Lambda_{n}^{i} u_{n}-p\right)\right\|^{2}\right) \\
\leq & \frac{1}{2}\left(\left\|\Lambda_{n}^{i-1} u_{n}-p\right\|^{2}+\left\|\Lambda_{n}^{i} u_{n}-p\right\|^{2}\right. \\
& \left.-\left\|\Lambda_{n}^{i-1} u_{n}-\Lambda_{n}^{i} u_{n}-\lambda_{i, n}\left(B_{i} \Lambda_{n}^{i-1} u_{n}-B_{i} p\right)\right\|^{2}\right) \\
\leq & \frac{1}{2}\left(\left\|u_{n}-p\right\|^{2}+\left\|\Lambda_{n}^{i} u_{n}-p\right\|^{2}\right. \\
& \left.-\left\|\Lambda_{n}^{i-1} u_{n}-\Lambda_{n}^{i} u_{n}-\lambda_{i, n}\left(B_{i} \Lambda_{n}^{i-1} u_{n}-B_{i} p\right)\right\|^{2}\right) \\
\leq & \frac{1}{2}\left(\left\|x_{n}-p\right\|^{2}+\left\|\Lambda_{n}^{i} u_{n}-p\right\|^{2}\right. \\
& \left.-\left\|\Lambda_{n}^{i-1} u_{n}-\Lambda_{n}^{i} u_{n}-\lambda_{i, n}\left(B_{i} \Lambda_{n}^{i-1} u_{n}-B_{i} p\right)\right\|^{2}\right)
\end{aligned}
$$

which immediately leads to

$$
\begin{aligned}
\left\|\Lambda_{n}^{i} u_{n}-p\right\|^{2} & \leq\left\|x_{n}-p\right\|^{2}-\left\|\Lambda_{n}^{i-1} u_{n}-\Lambda_{n}^{i} u_{n}-\lambda_{i, n}\left(B_{i} \Lambda_{n}^{i-1} u_{n}-B_{i} p\right)\right\|^{2} \\
& =\left\|x_{n}-p\right\|^{2}-\left\|\Lambda_{n}^{i-1} u_{n}-\Lambda_{n}^{k} u_{n}\right\|^{2}-\lambda_{i, n}^{2}\left\|B_{i} \Lambda_{n}^{i-1} u_{n}-B_{i} p\right\|^{2}
\end{aligned}
$$




$$
\begin{aligned}
& +2 \lambda_{i, n}\left|\Lambda_{n}^{i-1} u_{n}-\Lambda_{n}^{i} u_{n}, B_{i} \Lambda_{n}^{i-1} u_{n}-B_{i} p\right\rangle \\
\leq & \left\|x_{n}-p\right\|^{2}-\left\|\Lambda_{n}^{i-1} u_{n}-\Lambda_{n}^{i} u_{n}\right\|^{2} \\
& +2 \lambda_{i, n}\left\|\Lambda_{n}^{i-1} u_{n}-\Lambda_{n}^{i} u_{n}\right\|\left\|B_{i} \Lambda_{n}^{i-1} u_{n}-B_{i} p\right\| .
\end{aligned}
$$

Combining (3.18) and (3.23) we conclude that

$$
\begin{aligned}
\left\|z_{n}-p\right\|^{2} \leq & \beta_{n}\left\|x_{n}-p\right\|^{2}+\left(1-\beta_{n}\right)\left\|v_{n}-p\right\|^{2}+2 v_{n} \alpha_{n}\|p\|\left\|p-y_{n}\right\| \\
\leq & \beta_{n}\left\|x_{n}-p\right\|^{2}+\left(1-\beta_{n}\right)\left\|\Lambda_{n}^{i} u_{n}-p\right\|^{2}+2 v_{n} \alpha_{n}\|p\|\left\|p-y_{n}\right\| \\
\leq & \beta_{n}\left\|x_{n}-p\right\|^{2}+\left(1-\beta_{n}\right)\left[\left\|x_{n}-p\right\|^{2}-\left\|\Lambda_{n}^{i-1} u_{n}-\Lambda_{n}^{i} u_{n}\right\|^{2}\right. \\
& \left.+2 \lambda_{i, n}\left\|\Lambda_{n}^{i-1} u_{n}-\Lambda_{n}^{i} u_{n}\right\|\left\|B_{i} \Lambda_{n}^{i-1} u_{n}-B_{i} p\right\|\right]+2 v_{n} \alpha_{n}\|p\|\left\|p-y_{n}\right\| \\
\leq & \left\|x_{n}-p\right\|^{2}-\left(1-\beta_{n}\right)\left\|\Lambda_{n}^{i-1} u_{n}-\Lambda_{n}^{i} u_{n}\right\|^{2} \\
& +2 \lambda_{i, n}\left\|\Lambda_{n}^{i-1} u_{n}-\Lambda_{n}^{i} u_{n}\right\|\left\|B_{i} \Lambda_{n}^{i-1} u_{n}-B_{i} p\right\|+2 v_{n} \alpha_{n}\|p\|\left\|p-y_{n}\right\|,
\end{aligned}
$$

which yields

$$
\begin{aligned}
(1-d) & \left\|\Lambda_{n}^{i-1} u_{n}-\Lambda_{n}^{i} u_{n}\right\|^{2} \\
\leq & \left(1-\beta_{n}\right)\left\|\Lambda_{n}^{i-1} u_{n}-\Lambda_{n}^{i} u_{n}\right\|^{2} \\
\leq & \left\|x_{n}-p\right\|^{2}-\left\|z_{n}-p\right\|^{2}+2 \lambda_{i, n}\left\|\Lambda_{n}^{i-1} u_{n}-\Lambda_{n}^{i} u_{n}\right\|\left\|B_{i} \Lambda_{n}^{i-1} u_{n}-B_{i} p\right\| \\
& +2 v_{n} \alpha_{n}\|p\|\left\|p-y_{n}\right\| \\
\leq & \left\|x_{n}-z_{n}\right\|\left(\left\|x_{n}-p\right\|+\left\|z_{n}-p\right\|\right)+2 \lambda_{i, n}\left\|\Lambda_{n}^{i-1} u_{n}-\Lambda_{n}^{i} u_{n}\right\|\left\|B_{i} \Lambda_{n}^{i-1} u_{n}-B_{i} p\right\| \\
& +2 v_{n} \alpha_{n}\|p\|\left\|p-y_{n}\right\| .
\end{aligned}
$$

Since $\alpha_{n} \rightarrow 0,\left\{v_{n}\right\} \subset[\hat{a}, \hat{b}] \subset\left(0, \frac{1}{L}\right)$ and $\left\{\lambda_{i, n}\right\} \subset\left[a_{i}, b_{i}\right] \subset\left(0,2 \eta_{i}\right)$ where $i \in\{1,2, \ldots, N\}$, we deduce from (3.21) and the boundedness of $\left\{u_{n}\right\},\left\{x_{n}\right\},\left\{y_{n}\right\},\left\{z_{n}\right\}$ that

$$
\lim _{n \rightarrow \infty}\left\|\Lambda_{n}^{i-1} u_{n}-\Lambda_{n}^{i} u_{n}\right\|=0, \quad \forall i \in\{1,2, \ldots, N\}
$$

Also, combining (3.3), (3.18), and (3.22) we deduce that

$$
\begin{aligned}
\left\|z_{n}-p\right\|^{2} \leq & \beta_{n}\left\|x_{n}-p\right\|^{2}+\left(1-\beta_{n}\right)\left\|v_{n}-p\right\|^{2}+2 v_{n} \alpha_{n}\|p\|\left\|p-y_{n}\right\| \\
\leq & \beta_{n}\left\|x_{n}-p\right\|^{2}+\left(1-\beta_{n}\right)\left\|u_{n}-p\right\|^{2}+2 v_{n} \alpha_{n}\|p\|\left\|p-y_{n}\right\| \\
\leq & \beta_{n}\left\|x_{n}-p\right\|^{2}+\left(1-\beta_{n}\right)\left\|\Delta_{n}^{k} x_{n}-p\right\|^{2}+2 v_{n} \alpha_{n}\|p\|\left\|p-y_{n}\right\| \\
\leq & \beta_{n}\left\|x_{n}-p\right\|^{2}+\left(1-\beta_{n}\right)\left[\left\|x_{n}-p\right\|^{2}-\left\|\Delta_{n}^{k-1} x_{n}-\Delta_{n}^{k} x_{n}\right\|^{2}\right. \\
& \left.+2 r_{k, n}\left\|\Delta_{n}^{k-1} x_{n}-\Delta_{n}^{k} x_{n}\right\|\left\|A_{k} \Delta_{n}^{k-1} x_{n}-A_{k} p\right\|\right] \\
& +2 v_{n} \alpha_{n}\|p\|\left\|p-y_{n}\right\| \\
\leq & \left\|x_{n}-p\right\|^{2}-\left(1-\beta_{n}\right)\left\|\Delta_{n}^{k-1} x_{n}-\Delta_{n}^{k} x_{n}\right\|^{2} \\
& +2 r_{k, n}\left\|\Delta_{n}^{k-1} x_{n}-\Delta_{n}^{k} x_{n}\right\|\left\|A_{k} \Delta_{n}^{k-1} x_{n}-A_{k} p\right\| \\
& +2 v_{n} \alpha_{n}\|p\|\left\|p-y_{n}\right\|,
\end{aligned}
$$


which leads to

$$
\begin{aligned}
(1-d) & \left\|\Delta_{n}^{k-1} x_{n}-\Delta_{n}^{k} x_{n}\right\|^{2} \\
\leq & \left(1-\beta_{n}\right)\left\|\Delta_{n}^{k-1} x_{n}-\Delta_{n}^{k} x_{n}\right\|^{2} \\
\leq & \left\|x_{n}-p\right\|^{2}-\left\|z_{n}-p\right\|^{2}+2 r_{k, n}\left\|\Delta_{n}^{k-1} x_{n}-\Delta_{n}^{k} x_{n}\right\|\left\|A_{k} \Delta_{n}^{k-1} x_{n}-A_{k} p\right\| \\
& +2 v_{n} \alpha_{n}\|p\|\left\|p-y_{n}\right\| \\
\leq & \left\|x_{n}-z_{n}\right\|\left(\left\|x_{n}-p\right\|+\left\|z_{n}-p\right\|\right)+2 r_{k, n}\left\|\Delta_{n}^{k-1} x_{n}-\Delta_{n}^{k} x_{n}\right\|\left\|A_{k} \Delta_{n}^{k-1} x_{n}-A_{k} p\right\| \\
& +2 v_{n} \alpha_{n}\|p\|\left\|p-y_{n}\right\| .
\end{aligned}
$$

Since $\alpha_{n} \rightarrow 0,\left\{v_{n}\right\} \subset[\hat{a}, \hat{b}] \subset\left(0, \frac{1}{L}\right)$ and $\left\{r_{k, n}\right\} \subset\left[c_{k}, d_{k}\right] \subset\left(0,2 \mu_{k}\right)$ where $k \in\{1,2, \ldots, M\}$, we deduce from (3.21) and the boundedness of $\left\{x_{n}\right\},\left\{y_{n}\right\},\left\{z_{n}\right\}$ that

$$
\lim _{n \rightarrow \infty}\left\|\Delta_{n}^{k-1} x_{n}-\Delta_{n}^{k} x_{n}\right\|=0, \quad \forall k \in\{1,2, \ldots, M\}
$$

Hence from (3.24) and (3.25) we get

$$
\begin{aligned}
\left\|x_{n}-u_{n}\right\| & =\left\|\Delta_{n}^{0} x_{n}-\Delta_{n}^{M} x_{n}\right\| \\
& \leq\left\|\Delta_{n}^{0} x_{n}-\Delta_{n}^{1} x_{n}\right\|+\left\|\Delta_{n}^{1} x_{n}-\Delta_{n}^{2} x_{n}\right\|+\cdots+\left\|\Delta_{n}^{M-1} x_{n}-\Delta_{n}^{M} x_{n}\right\| \\
& \rightarrow 0 \quad \text { as } n \rightarrow \infty
\end{aligned}
$$

and

$$
\begin{aligned}
\left\|u_{n}-v_{n}\right\| & =\left\|\Lambda_{n}^{0} u_{n}-\Lambda_{n}^{N} u_{n}\right\| \\
& \leq\left\|\Lambda_{n}^{0} u_{n}-\Lambda_{n}^{1} u_{n}\right\|+\left\|\Lambda_{n}^{1} u_{n}-\Lambda_{n}^{2} u_{n}\right\|+\cdots+\left\|\Lambda_{n}^{N-1} u_{n}-\Lambda_{n}^{N} u_{n}\right\| \\
& \rightarrow 0 \quad \text { as } n \rightarrow \infty
\end{aligned}
$$

respectively. Thus, from (3.26) and (3.27) we obtain

$$
\left\|x_{n}-v_{n}\right\| \leq\left\|x_{n}-u_{n}\right\|+\left\|u_{n}-v_{n}\right\| \rightarrow 0 \quad \text { as } n \rightarrow \infty
$$

In addition, it is clear that

$$
\left\|x_{n}-W x_{n}\right\| \leq\left\|x_{n}-W_{n} x_{n}\right\|+\left\|W_{n} x_{n}-W x_{n}\right\| .
$$

Thus, we conclude from Remark 2.2, (3.15), and the boundedness of $\left\{x_{n}\right\}$ that

$$
\lim _{n \rightarrow \infty}\left\|x_{n}-W x_{n}\right\|=0
$$

Noting that $\left\|x_{n}-y_{n}\right\| \leq\left\|x_{n}-v_{n}\right\|+\left\|v_{n}-y_{n}\right\|$, we have from (3.13) and (3.28) that

$$
\lim _{n \rightarrow \infty}\left\|x_{n}-y_{n}\right\|=0
$$


Again, noting that $\left\|t_{n}-x_{n}\right\| \leq\left\|t_{n}-y_{n}\right\|+\left\|y_{n}-x_{n}\right\|$, we obtain from (3.17) and (3.30)

$$
\lim _{n \rightarrow \infty}\left\|t_{n}-x_{n}\right\|=0
$$

Furthermore, from (3.1) we find that $z_{n}-t_{n}=\beta_{n}\left(x_{n}-t_{n}\right)+\sigma_{n}\left(T t_{n}-t_{n}\right)$ and hence

$$
\begin{aligned}
\sigma_{n}\left(T t_{n}-t_{n}\right) & =z_{n}-t_{n}-\beta_{n}\left(x_{n}-t_{n}\right) \\
& =z_{n}-x_{n}+x_{n}-t_{n}-\beta_{n}\left(x_{n}-t_{n}\right) \\
& =z_{n}-x_{n}+\left(1-\beta_{n}\right)\left(x_{n}-t_{n}\right),
\end{aligned}
$$

which immediately leads to

$$
\sigma_{n}\left\|T t_{n}-t_{n}\right\| \leq\left\|z_{n}-x_{n}\right\|+\left(1-\beta_{n}\right)\left\|x_{n}-t_{n}\right\| \leq\left\|z_{n}-x_{n}\right\|+\left\|x_{n}-t_{n}\right\| .
$$

Consequently, from (3.14), (3.31), and $\liminf _{n \rightarrow \infty} \sigma_{n}>0$ we get

$$
\lim _{n \rightarrow \infty}\left\|T t_{n}-t_{n}\right\|=0
$$

Step 3. $\omega_{w}\left(x_{n}\right) \subset \Omega$.

Since $H$ is reflexive and $\left\{x_{n}\right\}$ is bounded, there exists at least a weak convergence subsequence of $\left\{x_{n}\right\}$. Hence it is well known that $\omega_{w}\left(x_{n}\right) \neq \emptyset$. Now, take an arbitrary $w \in \omega_{w}\left(x_{n}\right)$. Then there exists a subsequence $\left\{x_{n_{i}}\right\}$ of $\left\{x_{n}\right\}$ such that $x_{n_{i}} \rightarrow w$. From (3.24)-(3.26), (3.28), (3.30), and (3.31), we have $t_{n_{i}} \rightarrow w, y_{n_{i}} \rightarrow w, u_{n_{i}} \rightarrow w, v_{n_{i}} \rightarrow w, \Lambda_{n_{i}}^{m} u_{n_{i}} \rightarrow w$ and $\Delta_{n_{i}}^{k} x_{n_{i}} \rightarrow w$, where $m \in\{1,2, \ldots, N\}$ and $k \in\{1,2, \ldots, M\}$. Utilizing Proposition 2.4(ii) and Lemma 2.2, we deduce from (3.29) and (3.32) that $w \in \operatorname{Fix}(T)$ and $w \in \operatorname{Fix}(W)=$ $\bigcap_{n=1}^{\infty} \operatorname{Fix}\left(T_{n}\right)$ (due to Lemma 2.4). Next, we prove that $w \in \bigcap_{m=1}^{N} \mathrm{I}\left(B_{m}, R_{m}\right)$. As a matter of fact, since $B_{m}$ is $\eta_{m}$-inverse-strongly monotone, $B_{m}$ is a monotone and Lipschitzcontinuous mapping. It follows from Lemma 2.11 that $R_{m}+B_{m}$ is maximal monotone. Let $(v, g) \in G\left(R_{m}+B_{m}\right)$, i.e., $g-B_{m} v \in R_{m} v$. Again, since $\Lambda_{n}^{m} u_{n}=J_{R_{m}, \lambda_{m, n}}\left(I-\lambda_{m, n} B_{m}\right) \Lambda_{n}^{m-1} u_{n}$, $n \geq 1, m \in\{1,2, \ldots, N\}$, we have

$$
\Lambda_{n}^{m-1} u_{n}-\lambda_{m, n} B_{m} \Lambda_{n}^{m-1} u_{n} \in\left(I+\lambda_{m, n} R_{m}\right) \Lambda_{n}^{m} u_{n},
$$

that is,

$$
\frac{1}{\lambda_{m, n}}\left(\Lambda_{n}^{m-1} u_{n}-\Lambda_{n}^{m} u_{n}-\lambda_{m, n} B_{m} \Lambda_{n}^{m-1} u_{n}\right) \in R_{m} \Lambda_{n}^{m} u_{n}
$$

In terms of the monotonicity of $R_{m}$, we get

$$
\left\langle v-\Lambda_{n}^{m} u_{n}, g-B_{m} v-\frac{1}{\lambda_{m, n}}\left(\Lambda_{n}^{m-1} u_{n}-\Lambda_{n}^{m} u_{n}-\lambda_{m, n} B_{m} \Lambda_{n}^{m-1} u_{n}\right)\right\rangle \geq 0
$$

and hence

$$
\begin{aligned}
& \left\langle v-\Lambda_{n}^{m} u_{n}, g\right\rangle \\
& \quad \geq\left\langle v-\Lambda_{n}^{m} u_{n}, B_{m} v+\frac{1}{\lambda_{m, n}}\left(\Lambda_{n}^{m-1} u_{n}-\Lambda_{n}^{m} u_{n}-\lambda_{m, n} B_{m} \Lambda_{n}^{m-1} u_{n}\right)\right\rangle
\end{aligned}
$$




$$
\begin{aligned}
& =\left\langle v-\Lambda_{n}^{m} u_{n}, B_{m} v-B_{m} \Lambda_{n}^{m} u_{n}+B_{m} \Lambda_{n}^{m} u_{n}-B_{m} \Lambda_{n}^{m-1} u_{n}+\frac{1}{\lambda_{m, n}}\left(\Lambda_{n}^{m-1} u_{n}-\Lambda_{n}^{m} u_{n}\right)\right\rangle \\
& \geq\left\langle v-\Lambda_{n}^{m} u_{n}, B_{m} \Lambda_{n}^{m} u_{n}-B_{m} \Lambda_{n}^{m-1} u_{n}\right\rangle+\left\langle v-\Lambda_{n}^{m} u_{n}, \frac{1}{\lambda_{m, n}}\left(\Lambda_{n}^{m-1} u_{n}-\Lambda_{n}^{m} u_{n}\right)\right\rangle .
\end{aligned}
$$

In particular,

$$
\begin{aligned}
\left\langle v-\Lambda_{n_{i}}^{m} u_{n_{i}}, g\right\rangle \geq & \left\langle v-\Lambda_{n_{i}}^{m} u_{n_{i}}, B_{m} \Lambda_{n_{i}}^{m} u_{n_{i}}-B_{m} \Lambda_{n_{i}}^{m-1} u_{n_{i}}\right\rangle \\
& +\left\langle v-\Lambda_{n_{i}}^{m} u_{n_{i}}, \frac{1}{\lambda_{m, n_{i}}}\left(\Lambda_{n_{i}}^{m-1} u_{n_{i}}-\Lambda_{n_{i}}^{m} u_{n_{i}}\right)\right\rangle .
\end{aligned}
$$

Since $\left\|\Lambda_{n}^{m} u_{n}-\Lambda_{n}^{m-1} u_{n}\right\| \rightarrow 0$ (due to (3.24)) and $\left\|B_{m} \Lambda_{n}^{m} u_{n}-B_{m} \Lambda_{n}^{m-1} u_{n}\right\| \rightarrow 0$ (due to the Lipschitz continuity of $\left.B_{m}\right)$, we conclude from $\Lambda_{n_{i}}^{m} u_{n_{i}} \rightarrow w$ and $\left\{\lambda_{i, n}\right\} \subset\left[a_{i}, b_{i}\right] \subset\left(0,2 \eta_{i}\right)$ that

$$
\lim _{i \rightarrow \infty}\left\langle v-\Lambda_{n_{i}}^{m} u_{n_{i}}, g\right\rangle=\langle v-w, g\rangle \geq 0
$$

It follows from the maximal monotonicity of $B_{m}+R_{m}$ that $0 \in\left(R_{m}+B_{m}\right) w$, i.e., $w \in$ $\mathrm{I}\left(B_{m}, R_{m}\right)$. Therefore, $w \in \bigcap_{m=1}^{N} \mathrm{I}\left(B_{m}, R_{m}\right)$. Next we prove that $w \in \bigcap_{k=1}^{M} \operatorname{GMEP}\left(\Theta_{k}, \varphi_{k}, A_{k}\right)$. Since $\Delta_{n}^{k} x_{n}=T_{r_{k, n}}^{\left(\Theta_{k}, \varphi_{k}\right)}\left(I-r_{k, n} A_{k}\right) \Delta_{n}^{k-1} x_{n}, n \geq 1, k \in\{1,2, \ldots, M\}$, we have

$$
\begin{gathered}
\Theta_{k}\left(\Delta_{n}^{k} x_{n}, y\right)+\varphi_{k}(y)-\varphi_{k}\left(\Delta_{n}^{k} x_{n}\right)+\left\langle A_{k} \Delta_{n}^{k-1} x_{n}, y-\Delta_{n}^{k} x_{n}\right\rangle \\
+\frac{1}{r_{k, n}}\left\langle y-\Delta_{n}^{k} x_{n}, \Delta_{n}^{k} x_{n}-\Delta_{n}^{k-1} x_{n}\right\rangle \geq 0 .
\end{gathered}
$$

By (A2), we have

$$
\begin{aligned}
& \varphi_{k}(y)-\varphi_{k}\left(\Delta_{n}^{k} x_{n}\right)+\left\langle A_{k} \Delta_{n}^{k-1} x_{n}, y-\Delta_{n}^{k} x_{n}\right\rangle+\frac{1}{r_{k, n}}\left\langle y-\Delta_{n}^{k} x_{n}, \Delta_{n}^{k} x_{n}-\Delta_{n}^{k-1} x_{n}\right\rangle \\
& \geq \Theta_{k}\left(y, \Delta_{n}^{k} x_{n}\right) .
\end{aligned}
$$

Let $z_{t}=t y+(1-t) w$ for all $t \in(0,1]$ and $y \in C$. This implies that $z_{t} \in C$. Then we have

$$
\begin{aligned}
\left\langle z_{t}-\right. & \left.\Delta_{n}^{k} x_{n}, A_{k} z_{t}\right\rangle \\
\geq & \varphi_{k}\left(\Delta_{n}^{k} x_{n}\right)-\varphi_{k}\left(z_{t}\right)+\left\langle z_{t}-\Delta_{n}^{k} x_{n}, A_{k} z_{t}\right\rangle-\left\langle z_{t}-\Delta_{n}^{k} x_{n}, A_{k} \Delta_{n}^{k-1} x_{n}\right\rangle \\
& \quad-\left\langle z_{t}-\Delta_{n}^{k} x_{n}, \frac{\Delta_{n}^{k} x_{n}-\Delta_{n}^{k-1} x_{n}}{r_{k, n}}\right\rangle+\Theta_{k}\left(z_{t}, \Delta_{n}^{k} x_{n}\right) \\
= & \varphi_{k}\left(\Delta_{n}^{k} x_{n}\right)-\varphi_{k}\left(z_{t}\right)+\left\langle z_{t}-\Delta_{n}^{k} x_{n}, A_{k} z_{t}-A_{k} \Delta_{n}^{k} x_{n}\right\rangle \\
& +\left\langle z_{t}-\Delta_{n}^{k} x_{n}, A_{k} \Delta_{n}^{k} x_{n}-A_{k} \Delta_{n}^{k-1} x_{n}\right\rangle \\
& -\left\langle z_{t}-\Delta_{n}^{k} x_{n}, \frac{\Delta_{n}^{k} x_{n}-\Delta_{n}^{k-1} x_{n}}{r_{k, n}}\right\rangle+\Theta_{k}\left(z_{t}, \Delta_{n}^{k} x_{n}\right) .
\end{aligned}
$$

By (3.25), we have $\left\|A_{k} \Delta_{n}^{k} x_{n}-A_{k} \Delta_{n}^{k-1} x_{n}\right\| \rightarrow 0$ as $n \rightarrow \infty$. Furthermore, by the monotonicity of $A_{k}$, we obtain $\left\langle z_{t}-\Delta_{n}^{k} x_{n}, A_{k} z_{t}-A_{k} \Delta_{n}^{k} x_{n}\right\rangle \geq 0$. Then by (A4) we obtain

$$
\left\langle z_{t}-w, A_{k} z_{t}\right\rangle \geq \varphi_{k}(w)-\varphi_{k}\left(z_{t}\right)+\Theta_{k}\left(z_{t}, w\right)
$$


Utilizing (A1), (A4), and (3.34), we obtain

$$
\begin{aligned}
0 & =\Theta_{k}\left(z_{t}, z_{t}\right)+\varphi_{k}\left(z_{t}\right)-\varphi_{k}\left(z_{t}\right) \\
& \leq t \Theta_{k}\left(z_{t}, y\right)+(1-t) \Theta_{k}\left(z_{t}, w\right)+t \varphi_{k}(y)+(1-t) \varphi_{k}(w)-\varphi_{k}\left(z_{t}\right) \\
& \leq t\left[\Theta_{k}\left(z_{t}, y\right)+\varphi_{k}(y)-\varphi_{k}\left(z_{t}\right)\right]+(1-t)\left\langle z_{t}-w, A_{k} z_{t}\right\rangle \\
& =t\left[\Theta_{k}\left(z_{t}, y\right)+\varphi_{k}(y)-\varphi_{k}\left(z_{t}\right)\right]+(1-t) t\left\langle y-w, A_{k} z_{t}\right\rangle,
\end{aligned}
$$

and hence

$$
0 \leq \Theta_{k}\left(z_{t}, y\right)+\varphi_{k}(y)-\varphi_{k}\left(z_{t}\right)+(1-t)\left\langle y-w, A_{k} z_{t}\right\rangle
$$

Letting $t \rightarrow 0$, we have, for each $y \in C$,

$$
0 \leq \Theta_{k}(w, y)+\varphi_{k}(y)-\varphi_{k}(w)+\left\langle y-w, A_{k} w\right\rangle
$$

This implies that $w \in \operatorname{GMEP}\left(\Theta_{k}, \varphi_{k}, A_{k}\right)$ and hence $w \in \bigcap_{k=1}^{M} \operatorname{GMEP}\left(\Theta_{k}, \varphi_{k}, A_{k}\right)$. Furthermore, let us show that $w \in \operatorname{VI}(C, A)$. In fact, define

$$
\widetilde{T} v= \begin{cases}A v+N_{C} v, & \text { if } v \in C, \\ \emptyset, & \text { if } v \notin C,\end{cases}
$$

where $N_{C} v=\{u \in H:\langle v-x, u\rangle \geq 0, \forall x \in C\}$. Then $\widetilde{T}$ is maximal monotone and $0 \in \widetilde{T} v$ if and only if $v \in \operatorname{VI}(C, A)$; see [25]. Let $(v, \tilde{v}) \in G(\widetilde{T})$. Then we have $\tilde{v} \in \widetilde{T} v=A v+N_{C} v$, and hence $\tilde{v}-A v \in N_{C} v$. So, we have $\langle v-x, \tilde{v}-A v\rangle \geq 0$ for all $x \in C$. On the other hand, from $y_{n}=P_{C}\left(v_{n}-v_{n} A_{n} v_{n}\right)$ and $v \in C$, we get $\left\langle v_{n}-v_{n} A_{n} v_{n}-y_{n}, y_{n}-v\right\rangle \geq 0$, and hence,

$$
\left\langle v-y_{n}, \frac{y_{n}-v_{n}}{v_{n}}+A_{n} v_{n}\right\rangle \geq 0
$$

Therefore, from $\tilde{v}-A v \in N_{C} v$ and $y_{n_{i}} \in C$, we have

$$
\begin{aligned}
&\left\langle v-y_{n_{i}}, \tilde{v}\right\rangle \geq\left\langle v-y_{n_{i}}, A v\right\rangle \\
& \geq\left\langle v-y_{n_{i}}, A v\right\rangle-\left\langle v-y_{n_{i}}, \frac{y_{n_{i}}-v_{n_{i}}}{v_{n_{i}}}+A_{n_{i}} v_{n_{i}}\right\rangle \\
&=\left\langle v-y_{n_{i}}, A v\right\rangle-\left\langle v-y_{n_{i}}, \frac{y_{n_{i}}-v_{n_{i}}}{v_{n_{i}}}+A v_{n_{i}}\right\rangle-\alpha_{n_{i}}\left\langle v-y_{n_{i}}, v_{n_{i}}\right\rangle \\
&=\left\langle v-y_{n_{i}}, A v-A y_{n_{i}}\right\rangle+\left\langle v-y_{n_{i}}, A y_{n_{i}}-A v_{n_{i}}\right\rangle-\left\langle v-y_{n_{i}}, \frac{y_{n_{i}}-v_{n_{i}}}{v_{n_{i}}}\right\rangle \\
&-\alpha_{n_{i}}\left\langle v-y_{n_{i}}, v_{n_{i}}\right\rangle \\
& \geq\left\langle v-y_{n_{i}}, A y_{n_{i}}-A v_{n_{i}}\right\rangle-\left\langle v-y_{n_{i}}, \frac{y_{n_{i}}-v_{n_{i}}}{v_{n_{i}}}\right\rangle-\alpha_{n_{i}}\left\langle v-y_{n_{i}}, v_{n_{i}}\right\rangle .
\end{aligned}
$$

Hence, it is easy to see that $\langle v-w, \tilde{v}\rangle \geq 0$ as $i \rightarrow \infty$. Since $\widetilde{T}$ is maximal monotone, we have $w \in \widetilde{T}^{-1} 0$, and hence, $w \in \operatorname{VI}(C, A)$. Consequently, $w \in \bigcap_{n=1}^{\infty} \operatorname{Fix}\left(T_{n}\right) \cap$ 
$\bigcap_{k=1}^{M} \operatorname{GMEP}\left(\Theta_{k}, \varphi_{k}, A_{k}\right) \cap \bigcap_{i=1}^{N} \mathrm{I}\left(B_{i}, R_{i}\right) \cap \operatorname{VI}(C, A) \cap \operatorname{Fix}(T)=: \Omega$. This shows that $\omega_{w}\left(x_{n}\right) \subset \Omega$.

Step 4. $\left\{x_{n}\right\}$ converges strongly to a unique solution $x^{*} \in \Omega$ of SHVI (1.10).

Indeed, according to $\left\|x_{n+1}-x_{n}\right\| \rightarrow 0$, we can take a subsequence $\left\{x_{n_{i}}\right\}$ of $\left\{x_{n}\right\}$ satisfying

$$
\begin{aligned}
\limsup _{n \rightarrow \infty}\left\langle(\gamma V-\mu F) x^{*}, x_{n+1}-x^{*}\right\rangle & =\limsup _{n \rightarrow \infty}\left\langle(\gamma V-\mu F) x^{*}, x_{n}-x^{*}\right\rangle \\
& =\limsup _{i \rightarrow \infty}\left\langle(\gamma V-\mu F) x^{*}, x_{n_{i}}-x^{*}\right\rangle .
\end{aligned}
$$

Without loss of generality, we may further assume that $x_{n_{i}} \rightarrow \tilde{x}$; then $\tilde{x} \in \Omega$ due to Step 3 . Since $x^{*}$ is a solution of SHVI (1.10), we get

$$
\limsup _{n \rightarrow \infty}\left\langle(\gamma V-\mu F) x^{*}, x_{n+1}-x^{*}\right\rangle=\left\langle(\gamma V-\mu F) x^{*}, \tilde{x}-x^{*}\right\rangle \leq 0 .
$$

Repeating the argument of (3.35), we have

$$
\limsup _{n \rightarrow \infty}\left\langle(\gamma S-\mu F) x^{*}, x_{n+1}-x^{*}\right\rangle \leq 0
$$

From (3.1) and (3.9), it follows that (noticing that $x_{n+1}=P_{C} w_{n}$ and $0<\gamma \leq \tau$ )

$$
\begin{aligned}
& \left\|x_{n+1}-x^{*}\right\|^{2} \\
& =\left\langle w_{n}-x^{*}, x_{n+1}-x^{*}\right\rangle+\left\langle P_{C} w_{n}-w_{n}, P_{C} w_{n}-x^{*}\right\rangle \\
& \leq\left\langle w_{n}-x^{*}, x_{n+1}-x^{*}\right\rangle \\
& =\left\langle\left(I-\epsilon_{n} \mu F\right) z_{n}-\left(I-\epsilon_{n} \mu F\right) x^{*}, x_{n+1}-x^{*}\right\rangle+\delta_{n} \epsilon_{n} \gamma\left\langle V x_{n}-V x^{*}, x_{n+1}-x^{*}\right\rangle \\
& +\epsilon_{n}\left(1-\delta_{n}\right) \gamma\left\langle S x_{n}-S x^{*}, x_{n+1}-x^{*}\right\rangle+\delta_{n} \epsilon_{n}\left\langle(\gamma V-\mu F) x^{*}, x_{n+1}-x^{*}\right\rangle \\
& +\epsilon_{n}\left(1-\delta_{n}\right)\left\langle(\gamma S-\mu F) x^{*}, x_{n+1}-x^{*}\right\rangle \\
& \leq\left(1-\epsilon_{n} \tau\right)\left\|z_{n}-x^{*}\right\|\left\|x_{n+1}-x^{*}\right\|+\left[\delta_{n} \epsilon_{n} \gamma \rho+\epsilon_{n}\left(1-\delta_{n}\right) \gamma\right]\left\|x_{n}-x^{*}\right\|\left\|x_{n+1}-x^{*}\right\| \\
& +\delta_{n} \epsilon_{n}\left\langle(\gamma V-\mu F) x^{*}, x_{n+1}-x^{*}\right\rangle+\epsilon_{n}\left(1-\delta_{n}\right)\left\langle(\gamma S-\mu F) x^{*}, x_{n+1}-x^{*}\right\rangle \\
& \leq\left(1-\epsilon_{n} \tau\right) \frac{1}{2}\left(\left\|z_{n}-x^{*}\right\|^{2}+\left\|x_{n+1}-x^{*}\right\|^{2}\right) \\
& +\left[\delta_{n} \epsilon_{n} \gamma \rho+\epsilon_{n}\left(1-\delta_{n}\right) \gamma\right] \frac{1}{2}\left(\left\|x_{n}-x^{*}\right\|^{2}+\left\|x_{n+1}-x^{*}\right\|^{2}\right) \\
& +\delta_{n} \epsilon_{n}\left\langle(\gamma V-\mu F) x^{*}, x_{n+1}-x^{*}\right\rangle+\epsilon_{n}\left(1-\delta_{n}\right)\left\langle(\gamma S-\mu F) x^{*}, x_{n+1}-x^{*}\right\rangle \\
& \leq\left(1-\epsilon_{n} \tau\right) \frac{1}{2}\left[\left(\left\|x_{n}-x^{*}\right\|+\sqrt{2} v_{n} \alpha_{n}\left\|x^{*}\right\|\right)^{2}+\left\|x_{n+1}-x^{*}\right\|^{2}\right] \\
& +\left[\delta_{n} \epsilon_{n} \gamma \rho+\epsilon_{n}\left(1-\delta_{n}\right) \gamma\right] \frac{1}{2}\left(\left\|x_{n}-x^{*}\right\|^{2}+\left\|x_{n+1}-x^{*}\right\|^{2}\right) \\
& +\delta_{n} \epsilon_{n}\left\langle(\gamma V-\mu F) x^{*}, x_{n+1}-x^{*}\right\rangle+\epsilon_{n}\left(1-\delta_{n}\right)\left\langle(\gamma S-\mu F) x^{*}, x_{n+1}-x^{*}\right\rangle \\
& \leq\left(1-\epsilon_{n} \tau\right) \frac{1}{2}\left(\left\|x_{n}-x^{*}\right\|^{2}+\alpha_{n} \tilde{M}_{2}+\left\|x_{n+1}-x^{*}\right\|^{2}\right) \\
& +\left[\delta_{n} \epsilon_{n} \gamma \rho+\epsilon_{n}\left(1-\delta_{n}\right) \gamma\right] \frac{1}{2}\left(\left\|x_{n}-x^{*}\right\|^{2}+\left\|x_{n+1}-x^{*}\right\|^{2}\right)
\end{aligned}
$$




$$
\begin{aligned}
& +\delta_{n} \epsilon_{n}\left\langle(\gamma V-\mu F) x^{*}, x_{n+1}-x^{*}\right\rangle+\epsilon_{n}\left(1-\delta_{n}\right)\left\langle(\gamma S-\mu F) x^{*}, x_{n+1}-x^{*}\right\rangle \\
\leq & {\left[1-\delta_{n} \epsilon_{n} \gamma(1-\rho)\right] \frac{1}{2}\left(\left\|x_{n}-x^{*}\right\|^{2}+\left\|x_{n+1}-x^{*}\right\|^{2}\right)+\delta_{n} \epsilon_{n}\left\langle(\gamma V-\mu F) x^{*}, x_{n+1}-x^{*}\right\rangle } \\
& +\epsilon_{n}\left(1-\delta_{n}\right)\left\langle(\gamma S-\mu F) x^{*}, x_{n+1}-x^{*}\right\rangle+\alpha_{n} \widetilde{M}_{2},
\end{aligned}
$$

where $\widetilde{M}_{2}=\sup _{n \geq 1}\left\{2 v_{n}\left\|x^{*}\right\|\left(\sqrt{2}\left\|x_{n}-x^{*}\right\|+v_{n} \alpha_{n}\left\|x^{*}\right\|\right)\right\}<\infty$. It turns out that

$$
\begin{aligned}
& \left\|x_{n+1}-x^{*}\right\|^{2} \\
& \leq \frac{1-\delta_{n} \epsilon_{n} \gamma(1-\rho)}{1+\delta_{n} \epsilon_{n} \gamma(1-\rho)}\left\|x_{n}-x^{*}\right\|^{2}+\frac{2}{1+\delta_{n} \epsilon_{n} \gamma(1-\rho)}\left[\delta_{n} \epsilon_{n}\left\langle(\gamma V-\mu F) x^{*}, x_{n+1}-x^{*}\right\rangle\right. \\
& \left.\quad+\epsilon_{n}\left(1-\delta_{n}\right)\left((\gamma S-\mu F) x^{*}, x_{n+1}-x^{*}\right\rangle+\alpha_{n} \widetilde{M}_{2}\right] \\
& \leq \\
& \quad\left[1-\delta_{n} \epsilon_{n} \gamma(1-\rho)\right]\left\|x_{n}-x^{*}\right\|^{2}+\frac{2}{1+\delta_{n} \epsilon_{n} \gamma(1-\rho)}\left[\delta_{n} \epsilon_{n}\left|(\gamma V-\mu F) x^{*}, x_{n+1}-x^{*}\right\rangle\right. \\
& \left.\quad+\epsilon_{n}\left(1-\delta_{n}\right)\left((\gamma S-\mu F) x^{*}, x_{n+1}-x^{*}\right)\right]+2 \alpha_{n} \widetilde{M}_{2} \\
& =\left(1-s_{n}\right)\left\|x_{n}-x^{*}\right\|^{2}+s_{n} b_{n}+t_{n},
\end{aligned}
$$

where $t_{n}=2 \alpha_{n} \widetilde{M}_{2}, s_{n}=\delta_{n} \epsilon_{n} \gamma(1-\rho)$ and

$$
\begin{aligned}
b_{n}= & \frac{2}{\gamma(1-\rho)\left[1+\delta_{n} \epsilon_{n} \gamma(1-\rho)\right]}\left\langle(\gamma V-\mu F) x^{*}, x_{n+1}-x^{*}\right\rangle \\
& +\frac{2\left(1-\delta_{n}\right)}{\delta_{n} \gamma(1-\rho)\left[1+\delta_{n} \epsilon_{n} \gamma(1-\rho)\right]}\left\langle(\gamma S-\mu F) x^{*}, x_{n+1}-x^{*}\right\rangle .
\end{aligned}
$$

In terms of conditions (C5) and (C6), we conclude from $0<1-\rho \leq 1$ that $\left\{s_{n}\right\} \subset(0,1]$ and $\sum_{n=1}^{\infty} s_{n}=\infty$. Note that $\frac{2}{\gamma(1-\rho)\left[1+\delta_{n} \epsilon_{n} \gamma(1-\rho)\right]} \leq \frac{2}{\gamma(1-\rho)}$ and $\frac{2\left(1-\delta_{n}\right)}{\delta_{n} \gamma(1-\rho)\left[1+\delta_{n} \epsilon_{n} \gamma(1-\rho)\right]} \leq \frac{2}{a \gamma(1-\rho)}$, where $a=\inf \left\{\delta_{n}: n \geq 1\right\}>0$. Consequently, utilizing Lemma 2.13 we obtain

$$
\begin{aligned}
\limsup _{n \rightarrow \infty} b_{n} \leq & \limsup _{n \rightarrow \infty} \frac{2}{\gamma(1-\rho)\left[1+\delta_{n} \epsilon_{n} \gamma(1-\rho)\right]}\left\langle(\gamma V-\mu F) x^{*}, x_{n+1}-x^{*}\right\rangle \\
& +\limsup _{n \rightarrow \infty} \frac{2\left(1-\delta_{n}\right)}{\delta_{n} \gamma(1-\rho)\left[1+\delta_{n} \epsilon_{n} \gamma(1-\rho)\right]}\left\langle(\gamma S-\mu F) x^{*}, x_{n+1}-x^{*}\right\rangle \\
\leq & 0 .
\end{aligned}
$$

So, applying Lemma 2.12 to (3.37), we infer that $\lim _{n \rightarrow \infty}\left\|x_{n}-x^{*}\right\|=0$. The proof is complete.

Remark 3.1 In Theorem 3.1, whenever $V \equiv 0$, the iterative scheme (3.1) reduces to the following one:

$$
\left\{\begin{array}{l}
u_{n}=T_{r_{M, n}}^{\left(\Theta_{M}, \varphi_{M}\right)}\left(I-r_{M, n} A_{M}\right) T_{r_{M-1, n}}^{\left(\Theta_{M-1}, \varphi_{M-1}\right)}\left(I-r_{M-1, n} A_{M-1}\right) \cdots T_{r_{1, n}}^{\left(\Theta_{1}, \varphi_{1}\right)}\left(I-r_{1, n} A_{1}\right) x_{n}, \\
v_{n}=J_{R_{N}, \lambda_{N, n}}\left(I-\lambda_{N, n} B_{N}\right) J_{R_{N-1}, \lambda_{N-1, n}}\left(I-\lambda_{N-1, n} B_{N-1}\right) \cdots J_{R_{1}, \lambda_{1, n}}\left(I-\lambda_{1, n} B_{1}\right) u_{n}, \\
y_{n}=P_{C}\left(v_{n}-v_{n} A_{n} v_{n}\right) \\
z_{n}=\beta_{n} W_{n} x_{n}+\gamma_{n} P_{C}\left(v_{n}-v_{n} A_{n} y_{n}\right)+\sigma_{n} T P_{C}\left(v_{n}-v_{n} A_{n} y_{n}\right) \\
x_{n+1}=P_{C}\left[\epsilon_{n}\left(1-\delta_{n}\right) \gamma S x_{n}+\left(I-\epsilon_{n} \mu F\right) z_{n}\right], \quad \forall n \geq 1
\end{array}\right.
$$


where $A_{n}=\alpha_{n} I+A$ for all $n \geq 1$. Assume that the SHVI (1.11) has a solution and that all the conditions in Theorem 3.1 are satisfied. If $\left\{S x_{n}\right\}$ is bounded, then $\left\{x_{n}\right\}$ converges strongly to a unique solution of SHVI (1.11) provided $\lim _{n \rightarrow \infty}\left\|x_{n}-x_{n+1}\right\|=0$.

Next we consider a special case of SHVI (1.10). In SHVI (1.10), put $\mu=2, F=\frac{1}{2} I$ and $\gamma=\tau=1$. In this case, the objective is to find $x^{*} \in \Omega$ such that

$$
\left\{\begin{array}{l}
\left\langle(I-V) x^{*}, x-x^{*}\right\rangle \geq 0, \quad \forall x \in \Omega, \\
\left\langle(I-S) x^{*}, y-x^{*}\right\rangle \geq 0, \quad \forall y \in \Omega .
\end{array}\right.
$$

Utilizing Theorem 3.1 we immediately derive the following.

Corollary 3.1 Let $C$ be a nonempty closed convex subset of a real Hilbert space H. Let M, $N$ be two positive integers. Let $\Theta_{k}$ be a bifunction from $C \times C$ to $\mathbf{R}$ satisfying (A1)-(A4) and $\varphi_{k}: C \rightarrow \mathbf{R} \cup\{+\infty\}$ be a proper lower semicontinuous and convex function with restriction (B1) or (B2), where $k \in\{1,2, \ldots, M\}$. Let $R_{i}: C \rightarrow 2^{H}$ be a maximal monotone mapping and let $A_{k}: H \rightarrow H$ and $B_{i}: C \rightarrow H$ be $\mu_{k}$-inverse-strongly monotone and $\eta_{i}$-inverse-strongly monotone, respectively, where $k \in\{1,2, \ldots, M\}, i \in\{1,2, \ldots, N\}$. Let $\left\{T_{n}\right\}_{n=1}^{\infty}$ be a sequence of nonexpansive self-mappings on $C$ and $\left\{\lambda_{n}\right\}_{n=1}^{\infty}$ be a sequence in $(0, b]$ for some $b \in(0,1)$. Let $T: C \rightarrow C$ be a $\xi$-strictly pseudocontractive mapping, $S: C \rightarrow C$ be a nonexpansive mapping and $V: C \rightarrow H$ be a $\rho$-contraction with coefficient $\rho \in[0,1)$. Let $A: C \rightarrow H$ be a $\frac{1}{L}$-inverse-strongly monotone mapping. Assume that the SHVI (3.39) has a solution, where $\Omega:=\bigcap_{n=1}^{\infty} \operatorname{Fix}\left(T_{n}\right) \cap \bigcap_{k=1}^{M} \operatorname{GMEP}\left(\Theta_{k}, \varphi_{k}, A_{k}\right) \cap \bigcap_{i=1}^{N} \mathrm{I}\left(B_{i}, R_{i}\right) \cap \operatorname{VI}(C, A) \cap \operatorname{Fix}(T)$. Let $\left\{\alpha_{n}\right\} \subset$ $[0, \infty),\left\{v_{n}\right\} \subset\left(0, \frac{1}{L}\right),\left\{\epsilon_{n}\right\},\left\{\delta_{n}\right\},\left\{\beta_{n}\right\},\left\{\gamma_{n}\right\},\left\{\sigma_{n}\right\} \subset(0,1)$ and $\left\{\lambda_{i, n}\right\} \subset\left[a_{i}, b_{i}\right] \subset\left(0,2 \eta_{i}\right),\left\{r_{k, n}\right\} \subset$ $\left[c_{k}, d_{k}\right] \subset\left(0,2 \mu_{k}\right)$ where $i \in\{1,2, \ldots, N\}$ and $k \in\{1,2, \ldots, M\}$. For arbitrarily given $x_{1} \in C$, let $\left\{x_{n}\right\}$ be a sequence generated by

$$
\left\{\begin{array}{l}
u_{n}=T_{r_{M, n}}^{\left(\Theta_{M}, \varphi_{M}\right)}\left(I-r_{M, n} A_{M}\right) T_{r_{M-1, n}}^{\left(\Theta_{M-1}, \varphi_{M-1}\right)}\left(I-r_{M-1, n} A_{M-1}\right) \cdots T_{r_{1, n}}^{\left(\Theta_{1}, \varphi_{1}\right)}\left(I-r_{1, n} A_{1}\right) x_{n}, \\
v_{n}=J_{R_{N}, \lambda_{N, n}}\left(I-\lambda_{N, n} B_{N}\right) J_{R_{N-1}, \lambda_{N-1, n}}\left(I-\lambda_{N-1, n} B_{N-1}\right) \cdots J_{R_{1}, \lambda_{1, n}}\left(I-\lambda_{1, n} B_{1}\right) u_{n}, \\
y_{n}=P_{C}\left(v_{n}-v_{n} A_{n} v_{n}\right) \\
z_{n}=\beta_{n} W_{n} x_{n}+\gamma_{n} P_{C}\left(v_{n}-v_{n} A_{n} y_{n}\right)+\sigma_{n} T P_{C}\left(v_{n}-v_{n} A_{n} y_{n}\right) \\
x_{n+1}=P_{C}\left[\epsilon_{n}\left(\delta_{n} V x_{n}+\left(1-\delta_{n}\right) S x_{n}\right)+\left(1-\epsilon_{n}\right) z_{n}\right], \quad \forall n \geq 1,
\end{array}\right.
$$

where $A_{n}=\alpha_{n} I+A$ for all $n \geq 1$. Suppose that

(C1) $\sum_{n=1}^{\infty} \alpha_{n}<\infty$;

(C2) $0<\liminf _{n \rightarrow \infty} v_{n} \leq \limsup _{n \rightarrow \infty} v_{n}<\frac{1}{L}$;

(C3) $\beta_{n}+\gamma_{n}+\sigma_{n}=1$ and $\left(\gamma_{n}+\sigma_{n}\right) \xi \leq \gamma_{n}$ for all $n \geq 1$;

(C4) $0<\liminf _{n \rightarrow \infty} \beta_{n} \leq \lim \sup _{n \rightarrow \infty} \beta_{n}<1$ and $\liminf _{n \rightarrow \infty} \sigma_{n}>0$;

(C5) $0<\liminf _{n \rightarrow \infty} \delta_{n} \leq \limsup _{n \rightarrow \infty} \delta_{n}<1$;

(C6) $\lim _{n \rightarrow \infty} \epsilon_{n}=0$ and $\sum_{n=1}^{\infty} \epsilon_{n}=\infty$.

If $\left\{S x_{n}\right\}$ is bounded, then $\left\{x_{n}\right\}$ converges strongly to a unique solution of the SHVI (3.39) provided $\lim _{n \rightarrow \infty}\left\|x_{n}-x_{n+1}\right\|=0$.

In Theorem 3.1, putting $M=1$ and $N=2$, we obtain the following.

Corollary 3.2 Let $C$ be a nonempty closed convex subset of a real Hilbert space H. Let $\Theta_{1}$ be a bifunction from $C \times C$ to $\mathbf{R}$ satisfying (A1)-(A4), $\varphi_{1}: C \rightarrow \mathbf{R} \cup\{+\infty\}$ be a proper 
lower semicontinuous and convex function with restriction (B1) or (B2), and $A_{1}: H \rightarrow H$ be $\mu_{1}$-inverse strongly monotone. Let $R_{i}: C \rightarrow 2^{H}$ be a maximal monotone mapping and $B_{i}: C \rightarrow H$ be $\eta_{i}$-inverse-strongly monotone, for $i=1,2$. Let $\left\{T_{n}\right\}_{n=1}^{\infty}$ be a sequence of nonexpansive self-mappings on $C$ and $\left\{\lambda_{n}\right\}_{n=1}^{\infty}$ be a sequence in $(0, b]$ for some $b \in(0,1)$. Let $T: C \rightarrow C$ be a $\xi$-strictly pseudocontractive mapping, $S: C \rightarrow C$ be a nonexpansive mapping and $V: C \rightarrow H$ be a $\rho$-contraction with coefficient $\rho \in[0,1)$. Let $A: C \rightarrow H$ be a $\frac{1}{L}$-inverse-strongly monotone mapping, and $F: C \rightarrow H$ be $\kappa$-Lipschitzian and $\eta$-strongly monotone with positive constants $\kappa, \eta>0$ such that $0<\mu<\frac{2 \eta}{\kappa^{2}}$ and $0<\gamma \leq \tau$ where $\tau=1-$ $\sqrt{1-\mu\left(2 \eta-\mu \kappa^{2}\right)}$. Assume that the SHVI (1.10) has a solution, where $\Omega:=\bigcap_{n=1}^{\infty} \operatorname{Fix}\left(T_{n}\right) \cap$ $\operatorname{GMEP}\left(\Theta_{1}, \varphi_{1}, A_{1}\right) \cap \mathrm{I}\left(B_{2}, R_{2}\right) \cap \mathrm{I}\left(B_{1}, R_{1}\right) \cap \mathrm{VI}(C, A) \cap \operatorname{Fix}(T)$. Let $\left\{\alpha_{n}\right\} \subset[0, \infty),\left\{v_{n}\right\} \subset\left(0, \frac{1}{L}\right)$, $\left\{\epsilon_{n}\right\},\left\{\delta_{n}\right\},\left\{\beta_{n}\right\},\left\{\gamma_{n}\right\},\left\{\sigma_{n}\right\} \subset(0,1),\left\{r_{1, n}\right\} \subset\left[c_{1}, d_{1}\right] \subset\left(0,2 \mu_{1}\right)$ and $\left\{\lambda_{i, n}\right\} \subset\left[a_{i}, b_{i}\right] \subset\left(0,2 \eta_{i}\right)$ for $i=1$, 2. For arbitrarily given $x_{1} \in C$, let $\left\{x_{n}\right\}$ be a sequence generated by

$$
\left\{\begin{array}{l}
\Theta_{1}\left(u_{n}, y\right)+\varphi_{1}(y)-\varphi_{1}\left(u_{n}\right)+\left\langle A_{1} x_{n}, y-u_{n}\right\rangle+\frac{1}{r_{1, n}}\left\langle u_{n}-x_{n}, y-u_{n}\right\rangle \geq 0, \quad \forall y \in C, \\
v_{n}=J_{R_{2}, \lambda_{2, n}}\left(I-\lambda_{2, n} B_{2}\right) J_{R_{1}, \lambda_{1, n}}\left(I-\lambda_{1, n} B_{1}\right) u_{n}, \\
y_{n}=P_{C}\left(v_{n}-v_{n} A_{n} v_{n}\right), \\
z_{n}=\beta_{n} W_{n} x_{n}+\gamma_{n} P_{C}\left(v_{n}-v_{n} A_{n} y_{n}\right)+\sigma_{n} T P_{C}\left(v_{n}-v_{n} A_{n} y_{n}\right), \\
x_{n+1}=P_{C}\left[\epsilon_{n} \gamma\left(\delta_{n} V x_{n}+\left(1-\delta_{n}\right) S x_{n}\right)+\left(I-\epsilon_{n} \mu F\right) z_{n}\right], \quad \forall n \geq 1,
\end{array}\right.
$$

where $A_{n}=\alpha_{n} I+A$ for all $n \geq 1$. Suppose that

(C1) $\sum_{n=1}^{\infty} \alpha_{n}<\infty$;

(C2) $0<\liminf _{n \rightarrow \infty} v_{n} \leq \limsup _{n \rightarrow \infty} v_{n}<\frac{1}{L}$;

(C3) $\beta_{n}+\gamma_{n}+\sigma_{n}=1$ and $\left(\gamma_{n}+\sigma_{n}\right) \xi \leq \gamma_{n}$ for all $n \geq 1$;

(C4) $0<\liminf _{n \rightarrow \infty} \beta_{n} \leq \limsup \sup _{n \rightarrow \infty} \beta_{n}<1$ and $\liminf _{n \rightarrow \infty} \sigma_{n}>0$;

(C5) $0<\liminf _{n \rightarrow \infty} \delta_{n} \leq \limsup _{n \rightarrow \infty} \delta_{n}<1$;

(C6) $\lim _{n \rightarrow \infty} \epsilon_{n}=0$ and $\sum_{n=1}^{\infty} \epsilon_{n}=\infty$.

If $\left\{S x_{n}\right\}$ is bounded, then $\left\{x_{n}\right\}$ converges strongly to a unique solution of the SHVI (1.10) provided $\lim _{n \rightarrow \infty}\left\|x_{n}-x_{n+1}\right\|=0$.

In Theorem 3.1, putting $M=N=1$, we obtain the following.

Corollary 3.3 Let $C$ be a nonempty closed convex subset of a real Hilbert space H. Let $\Theta_{1}$ be a bifunction from $C \times C$ to $\mathbf{R}$ satisfying (A1)-(A4), $\varphi_{1}: C \rightarrow \mathbf{R} \cup\{+\infty\}$ be a proper lower semicontinuous and convex function with restriction (B1) or (B2), and $A_{1}: H \rightarrow H$ be $\mu_{1}$-inverse strongly monotone. Let $R_{1}: C \rightarrow 2^{H}$ be a maximal monotone mapping and $B_{1}: C \rightarrow H$ be $\eta_{1}$-inverse-strongly monotone. Let $\left\{T_{n}\right\}_{n=1}^{\infty}$ be a sequence of nonexpansive selfmappings on $C$ and $\left\{\lambda_{n}\right\}_{n=1}^{\infty}$ be a sequence in $(0, b]$ for some $b \in(0,1)$. Let $T: C \rightarrow C$ be a $\xi$-strictly pseudocontractive mapping, $S: C \rightarrow C$ be a nonexpansive mapping and $V: C \rightarrow$ $H$ be a $\rho$-contraction with coefficient $\rho \in[0,1)$. Let $A: C \rightarrow H$ be a $\frac{1}{L}$-inverse-strongly monotone mapping, and $F: C \rightarrow H$ be $\kappa$-Lipschitzian and $\eta$-strongly monotone with positive constants $\kappa, \eta>0$ such that $0<\mu<\frac{2 \eta}{\kappa^{2}}$ and $0<\gamma \leq \tau$ where $\tau=1-\sqrt{1-\mu\left(2 \eta-\mu \kappa^{2}\right)}$. Assume that the SHVI (1.10) has a solution, where $\Omega:=\bigcap_{n=1}^{\infty} \operatorname{Fix}\left(T_{n}\right) \cap \operatorname{GMEP}\left(\Theta_{1}, \varphi_{1}, A_{1}\right) \cap$ $\mathrm{I}\left(B_{1}, R_{1}\right) \cap \operatorname{VI}(C, A) \cap \operatorname{Fix}(T)$. Let $\left\{\alpha_{n}\right\} \subset[0, \infty),\left\{v_{n}\right\} \subset\left(0, \frac{1}{L}\right),\left\{\epsilon_{n}\right\},\left\{\delta_{n}\right\},\left\{\beta_{n}\right\},\left\{\gamma_{n}\right\},\left\{\sigma_{n}\right\} \subset$ $(0,1),\left\{\lambda_{1, n}\right\} \subset\left[a_{1}, b_{1}\right] \subset\left(0,2 \eta_{1}\right)$ and $\left\{r_{1, n}\right\} \subset\left[c_{1}, d_{1}\right] \subset\left(0,2 \mu_{1}\right)$. For arbitrarily given $x_{1} \in C$, 
let $\left\{x_{n}\right\}$ be a sequence generated by

$$
\left\{\begin{array}{l}
\Theta_{1}\left(u_{n}, y\right)+\varphi_{1}(y)-\varphi_{1}\left(u_{n}\right)+\left\langle A_{1} x_{n}, y-u_{n}\right\rangle+\frac{1}{r_{1, n}}\left\langle u_{n}-x_{n}, y-u_{n}\right\rangle \geq 0, \quad \forall y \in C, \\
v_{n}=J_{R_{1}, \lambda_{1, n}}\left(I-\lambda_{1, n} B_{1}\right) u_{n}, \\
y_{n}=P_{C}\left(v_{n}-v_{n} A_{n} v_{n}\right), \\
z_{n}=\beta_{n} W_{n} x_{n}+\gamma_{n} P_{C}\left(v_{n}-v_{n} A_{n} y_{n}\right)+\sigma_{n} T P_{C}\left(v_{n}-v_{n} A_{n} y_{n}\right), \\
x_{n+1}=P_{C}\left[\epsilon_{n} \gamma\left(\delta_{n} V x_{n}+\left(1-\delta_{n}\right) S x_{n}\right)+\left(I-\epsilon_{n} \mu F\right) z_{n}\right], \quad \forall n \geq 1,
\end{array}\right.
$$

where $A_{n}=\alpha_{n} I+A$ for all $n \geq 1$. Suppose that

(C1) $\sum_{n=1}^{\infty} \alpha_{n}<\infty$;

(C2) $0<\liminf _{n \rightarrow \infty} v_{n} \leq \limsup _{n \rightarrow \infty} v_{n}<\frac{1}{L}$;

(C3) $\beta_{n}+\gamma_{n}+\sigma_{n}=1$ and $\left(\gamma_{n}+\sigma_{n}\right) \xi \leq \gamma_{n}$ for all $n \geq 1$;

(C4) $0<\liminf _{n \rightarrow \infty} \beta_{n} \leq \limsup _{n \rightarrow \infty} \beta_{n}<1$ and $\liminf _{n \rightarrow \infty} \sigma_{n}>0$;

(C5) $0<\liminf _{n \rightarrow \infty} \delta_{n} \leq \limsup _{n \rightarrow \infty} \delta_{n}<1$;

(C6) $\lim _{n \rightarrow \infty} \epsilon_{n}=0$ and $\sum_{n=1}^{\infty} \epsilon_{n}=\infty$.

If $\left\{S x_{n}\right\}$ is bounded, then $\left\{x_{n}\right\}$ converges strongly to a unique solution of the SHVI (1.10) provided $\lim _{n \rightarrow \infty}\left\|x_{n}-x_{n+1}\right\|=0$.

Remark 3.2 It is obvious that our iterative scheme (3.1) is very different from Yao et al.'s iterative one (1.5) and Kong et al.'s iterative one (1.8). Here, the three-step iterative scheme in [7, Algorithm I] is extended to develop our five-step iterative scheme (3.1) for the SHVI (1.10) by combining Korpelevich's extragradient method, viscosity approximation method, hybrid steepest-descent method [48], Mann's iteration method and projection method. It is worth pointing out that under the lack of the assumptions similar to those in [31, Theorem 3.2], e.g., $\left\{x_{n}\right\}$ is bounded and $\operatorname{Fix}(T) \cap \operatorname{int} C \neq \emptyset$, the sequence $\left\{x_{n}\right\}$ generated by (3.1) converges strongly to a point $x^{*} \in \bigcap_{n=1}^{\infty} \operatorname{Fix}\left(T_{n}\right) \cap \bigcap_{k=1}^{M} \operatorname{GMEP}\left(\Theta_{k}, \varphi_{k}, A_{k}\right) \cap \bigcap_{i=1}^{N} \mathrm{I}\left(B_{i}, R_{i}\right) \cap$ $\operatorname{VI}(C, A) \cap \operatorname{Fix}(T)=: \Omega$, which is a unique solution $x^{*} \in \Omega$ of the SHVI (1.10) (over the fixed point set of an infinite family of nonexpansive mappings $\left\{T_{n}\right\}_{n=1}^{\infty}$ and a $\xi$-strictly pseudocontractive mapping $T$ ). It is worth emphasizing that the nonexpansive mapping $T$ in (1.5) is extended to a $\xi$-strictly pseudocontractive mapping $T$ in (3.1) and the VIP in SHVI (1.6) is extended to the setting of finitely many GMEPs and finitely many variational inclusions in SHVI (1.10).

Remark 3.3 Our Theorem 3.1 improves, extends, supplements and develops Yao et al. [31, Theorems 3.1 and 3.2] and Kong et al. [7, Theorem 17] in the following aspects:

(a) Our SHVI (1.10) with the unique solution $x^{*} \in \Omega$ satisfying

$$
x^{*}=P_{\bigcap_{n=1}^{\infty} \operatorname{Fix}\left(T_{n}\right) \cap \bigcap_{k=1}^{M} \operatorname{GMEP}\left(\Theta_{k}, \varphi_{k}, A_{k}\right) \cap \bigcap_{i=1}^{N} \mathrm{I}\left(B_{i}, R_{i}\right) \cap \mathrm{VI}(C, A) \cap \operatorname{Fix}(T)}(I-(\mu F-\gamma S)) x^{*}
$$

is more general than the problem of finding a point $\tilde{x} \in C$ satisfying $\tilde{x}=P_{\operatorname{Fix}(T)} S \tilde{x}$ in [31] and than the problem of finding a point $x^{*} \in \operatorname{Fix}(T) \cap \operatorname{VI}(C, A)$ satisfying $x^{*}=P_{\operatorname{Fix}(T) \cap V I(C, A)}(I-(\mu F-\gamma S)) x^{*}$ in [7, Theorem 17]. It is worth emphasizing that $S$ is nonexpansive if and only if the complement $I-S$ is $\frac{1}{2}$-inverse-strongly monotone; see [19].

(b) Our five-step iterative scheme (3.1) for SHVI (1.10) is more flexible, more advantageous and more subtle than Kong et al.'s three-step iterative one in 
[7, Algorithm I] and than Yao et al.'s two-step iterative one (1.5) because it can be used to solve several kinds of problems, e.g., the SHVI, the HVIP and the problem of finding a common point of four sets: $\bigcap_{n=1}^{\infty} \operatorname{Fix}\left(T_{n}\right) \cap \operatorname{Fix}(T)$, $\bigcap_{k=1}^{M} \operatorname{GMEP}\left(\Theta_{k}, \varphi_{k}, A_{k}\right), \bigcap_{i=1}^{N} \mathrm{I}\left(B_{i}, R_{i}\right)$ and $\operatorname{VI}(C, A)$. In addition, our Theorem 3.1 drops the crucial requirements in [31, Theorem 3.2] that $\lim _{n \rightarrow \infty} \frac{\alpha_{n}}{\beta_{n}}=0$, $\lim _{n \rightarrow \infty} \frac{\beta_{n}^{2}}{\alpha_{n}}=0, \operatorname{Fix}(T) \cap \operatorname{int} C \neq \emptyset$ and $\left\{x_{n}\right\}$ is bounded, generalizes [7, Theorem 17] from one nonlinear mapping $T$ to an infinite family of nonlinear mappings $\left\{T_{n}\right\}_{n=1}^{\infty}$ and $T$ and extends [7, Theorem 17] to the setting of finitely many GMEPs and finitely many variational inclusions.

(c) The argument techniques in our Theorem 3.1 are very different from the argument ones in [31, Theorems 3.1 and 3.2] and from the argument ones in [7, Theorem 17] because we make use of the properties of the $W$-mappings $W_{n}$ (see Lemmas 2.4 and 2.5), the properties of resolvent operators and maximal monotone mappings (see Proposition 2.4 and Lemmas 2.9-2.13), the inclusion problem $0 \in \widetilde{T} v$ ( $\Leftrightarrow v \in \mathrm{VI}(C, A)$ for maximal monotone operator $\widetilde{T}$ ) (see (2.3)) and the contractive coefficient estimates for the contractions associating with nonexpansive mappings (see Lemma 2.7).

\section{Competing interests}

The authors declare that they have no competing interests.

\section{Authors' contributions}

All authors contributed equally and significantly in writing this article. All authors read and approved the final manuscript.

\section{Author details}

${ }^{1}$ Department of Mathematics, Shanghai Normal University, Shanghai, 200234, China. ${ }^{2}$ Scientific Computing Key Laboratory of Shanghai Universities, Shanghai, 200234, China. ${ }^{3}$ Department of Mathematics, Banaras Hindu University, Varanasi, 221005, India. ${ }^{4}$ Center for Fundamental Science, Kaohsiung Medical University, Kaohsiung, 807, Taiwan.

${ }^{5}$ Department of Mathematics, King Abdulaziz University, P.O. Box 80203, Jeddah, 21589, Saudi Arabia.

\section{Acknowledgements}

Lu-Chuan Ceng was partially supported by the National Science Foundation of China (11071169), Innovation Program of Shanghai Municipal Education Commission and PhD Program Foundation of Ministry of Education of China (20123127110002). Jen-Chih Yao was partially supported by the grant NSC 102-2115-M-037-002-MY3.

Received: 3 July 2014 Accepted: 25 September 2014 Published: 19 Nov 2014

\section{References}

1. Lions, JL: Quelques Méthodes de Résolution des Problèmes aux Limites Non Linéaires. Dunod, Paris (1969)

2. Glowinski, R: Numerical Methods for Nonlinear Variational Problems. Springer, New York (1984)

3. Takahashi, W: Nonlinear Functional Analysis. Yokohama Publishers, Yokohama (2000)

4. Oden, JT: Quantitative Methods on Nonlinear Mechanics. Prentice Hall, Englewood Cliffs (1986)

5. Zeidler, E: Nonlinear Functional Analysis and Its Applications. Springer, New York (1985)

6. Korpelevich, GM: The extragradient method for finding saddle points and other problems. Matecon 12, 747-756 (1976)

7. Kong, ZR, Ceng, LC, Ansari, QH, Pang, CT: Multistep hybrid extragradient method for triple hierarchical variational inequalities. Abstr. Appl. Anal. 2013, Article ID 718624 (2013)

8. Zeng, LC, Yao, JC: Strong convergence theorem by an extragradient method for fixed point problems and variational inequality problems. Taiwan. J. Math. 10(5), 1293-1303 (2006)

9. Peng, JW, Yao, JC: A new hybrid-extragradient method for generalized mixed equilibrium problems, fixed point problems and variational inequality problems. Taiwan. J. Math. 12, 1401-1432 (2008)

10. Ceng, LC, Ansari, QH, Schaible, S: Hybrid extragradient-like methods for generalized mixed equilibrium problems, system of generalized equilibrium problems and optimization problems. J. Glob. Optim. 53, 69-96 (2012)

11. Ceng, LC, Ansari, QH, Wong, MM, Yao, JC: Mann type hybrid extragradient method for variational inequalities, variational inclusions and fixed point problems. Fixed Point Theory 13(2), 403-422 (2012)

12. Nadezhkina, N, Takahashi, W: Weak convergence theorem by an extragradient method for nonexpansive mappings and monotone mappings. J. Optim. Theory Appl. 128, 191-201 (2006)

13. Ceng, LC, Hadjisavvas, N, Wong, NC: Strong convergence theorem by a hybrid extragradient-like approximation method for variational inequalities and fixed point problems. J. Glob. Optim. 46, 635-646 (2010) 
14. Ceng, LC, Yao, JC: A relaxed extragradient-like method for a generalized mixed equilibrium problem, a general system of generalized equilibria and a fixed point problem. Nonlinear Anal. 72, 1922-1937 (2010)

15. Ceng, LC, Ansari, QH, Yao, JC: Relaxed extragradient iterative methods for variational inequalities. Appl. Math. Comput. 218, 1112-1123 (2011)

16. Ceng, LC, Petrusel, A: Relaxed extragradient-like method for general system of generalized mixed equilibria and fixed point problem. Taiwan. J. Math. 16(2), 445-478 (2012)

17. Ceng, LC, Guu, SM, Yao, JC: Hybrid iterative method for finding common solutions of generalized mixed equilibrium and fixed point problems. Fixed Point Theory Appl. 2012, 92 (2012)

18. Ceng, LC, Hu, HY, Wong, MM: Strong and weak convergence theorems for generalized mixed equilibrium problem with perturbation and fixed point problem of infinitely many nonexpansive mappings. Taiwan. J. Math. 15(3), 1341-1367 (2011)

19. Ceng, LC, Al-Homidan, S: Algorithms of common solutions for generalized mixed equilibria, variational inclusions, and constrained convex minimization. Abstr. Appl. Anal. 2014, Article ID 132053 (2014)

20. Takahashi, S, Takahashi, W: Strong convergence theorem for a generalized equilibrium problem and a nonexpansive mapping in a Hilbert space. Nonlinear Anal. 69, 1025-1033 (2008)

21. Ceng, LC, Yao, JC: A hybrid iterative scheme for mixed equilibrium problems and fixed point problems. J. Comput. Appl. Math. 214, 186-201 (2008)

22. Ceng, LC, Yao, JC: Hybrid viscosity approximation schemes for equilibrium problems and fixed point problems of infinitely many nonexpansive mappings. Appl. Math. Comput. 198, 729-741 (2008)

23. Colao, V, Marino, G, Xu, HK: An iterative method for finding common solutions of equilibrium and fixed point problems. J. Math. Anal. Appl. 344, 340-352 (2008)

24. Ceng, LC, Petrusel, A, Yao, JC: Iterative approaches to solving equilibrium problems and fixed point problems of infinitely many nonexpansive mappings. J. Optim. Theory Appl. 143, 37-58 (2009)

25. Rockafellar, RT: Monotone operators and the proximal point algorithms. SIAM J. Control Optim. 14, 877-898 (1976)

26. Huang, $\mathrm{NJ}$ : A new completely general class of variational inclusions with noncompact valued mappings. Comput. Math. Appl. 35(10), 9-14 (1998)

27. Zeng, LC, Guu, SM, Yao, JC: Characterization of $\mathrm{H}$-monotone operators with applications to variational inclusions. Comput. Math. Appl. 50(3-4), 329-337 (2005)

28. Ceng, LC, Guu, SM, Yao, JC: Iterative approximation of solutions for a class of completely generalized set-valued quasi-variational inclusions. Comput. Math. Appl. 56, 978-987 (2008)

29. Zhang, SS, Lee, HWJ, Chan, CK: Algorithms of common solutions for quasivariational inclusions and fixed point problems. Appl. Math. Mech. 29, 571-581 (2008)

30. Ceng, LC, Guu, SM, Yao, JC: Iterative algorithm for finding approximate solutions of mixed quasi-variational-like inclusions. Comput. Math. Appl. 56, 942-952 (2008)

31. Yao, Y, Liou, YC, Marino, G: Two-step iterative algorithms for hierarchical fixed point problems and variational inequality problems. J. Appl. Math. Comput. 31(1-2), 433-445 (2009)

32. Ceng, LC, Yao, JC: On the triple hierarchical variational inequalities with constraints of mixed equilibria, variational inclusions and systems of generalized equilibria. Tamkang J. Math. 45(3), 297-334 (2014)

33. Byrne, C: A unified treatment of some iterative algorithms in signal processing and image reconstruction. Inverse Probl. 20(1), 103-120 (2004)

34. Combettes, PL: Solving monotone inclusions via compositions of nonexpansive averaged operators. Optimization 53(5-6), 475-504 (2004)

35. Geobel, K, Kirk, WA: Topics on Metric Fixed Point Theory. Cambridge University Press, Cambridge (1990)

36. Shimoji, K, Takahashi, W: Strong convergence to common fixed points of infinite nonexpansive mappings and applications. Taiwan. J. Math. 5, 387-404 (2001)

37. Yao, Y, Liou, YC, Yao, JC: Convergence theorem for equilibrium problems and fixed point problems of infinite family of nonexpansive mappings. Fixed Point Theory Appl. 2007, Article ID 64363 (2007)

38. Marino, G, Xu, HK: Weak and strong convergence theorems for strict pseudo-contractions in Hilbert spaces. J. Math Anal. Appl. 329, 336-346 (2007)

39. Sahu, DR, Xu, HK, Yao, JC: Asymptotically strict pseudocontractive mappings in the intermediate sense. Nonlinear Anal. 70, 3502-3511 (2009)

40. Yao, Y, Liou, YC, Kang, SM: Approach to common elements of variational inequality problems and fixed point problems via a relaxed extragradient method. Comput. Math. Appl. 59, 3472-3480 (2010)

41. Han, D, Lo, HK: Solving non-additive traffic assignment problems: a descent method for co-coercive variational inequalities. Eur. J. Oper. Res. 159(3), 529-544 (2004)

42. Bertsekas, DP, Gafni, EM: Projection methods for variational inequalities with application to the traffic assignment problem. Math. Program. Stud. 17, 139-159 (1982)

43. $\mathrm{Xu}, \mathrm{HK}, \mathrm{Kim}, \mathrm{TH}$ : Convergence of hybrid steepest-descent methods for variational inequalities. J. Optim. Theory Appl. $119(1), 185-201(2003)$

44. Barbu, V: Nonlinear Semigroups and Differential Equations in Banach Spaces. Noordhoff, Groningen (1976)

45. Xu, HK: Iterative algorithms for nonlinear operators. J. Lond. Math. Soc. (2) 66(1), 240-256 (2002)

46. Ceng, LC, Petrusel, A, Yao, JC: Strong convergence theorems of averaging iterations of nonexpansive nonself-mappings in Banach spaces. Fixed Point Theory 8(2), 219-236 (2007)

47. Ceng, LC, Petrusel, A, Yao, JC: Relaxed extragradient methods with regularization for general system of variational inequalities with constraints of split feasibility and fixed point problems. Abstr. Appl. Anal. 2013, Article ID 891232 (2013)

48. Yamada, I: The hybrid steepest-descent method for the variational inequality problems over the intersection of the fixed-point sets of nonexpansive mappings. In: Batnariu, D, Censor, Y, Reich, S (eds.) Inherently Parallel Algorithms in Feasibility and Optimization and Their Applications, pp. 473-504. North-Holland, Amsterdam (2001)

10.1186/1029-242X-2014-460

Cite this article as: Ceng et al.: A unified extragradient method for systems of hierarchical variational inequalities in a

Hilbert space. Journal of Inequalities and Applications 2014, 2014:460 